\title{
The calculus of thermodynamical formalism
}

\author{
Paolo Giuliettii ${ }^{\mathrm{i}}$, Benoit R. Kloecknerii ${ }^{\mathrm{ii}}$, Artur O. Lopes ${ }^{\mathrm{iii}}$, \\ and Diego Marconiv§ \\ $\S$ Universidade Federal do Rio Grande do Sul, IME, 91509-900, Porto Alegre, Brazil \\ ‡Université Paris-Est, Laboratoire d’Analyse et de Matématiques Appliquées (UMR 8050), \\ UPEM, UPEC, CNRS, F-94010, Créteil, France
}

August 7, 2015

Given a finite-to-one map $T$ acting on a compact metric space $\Omega$ and an appropriate Banach space of functions $\mathcal{X}(\Omega)$, one classically constructs for each potential $A \in \mathcal{X}$ a transfer operator $\mathscr{L}_{A}$ acting on $\mathcal{X}(\Omega)$. Under suitable hypotheses, it is well-known that $\mathscr{L}_{A}$ has a maximal eigenvalue $\lambda_{A}$, has a spectral gap and defines a unique Gibbs measure $\mu_{A}$. Moreover there is a unique normalized potential of the form $B:=A+f-f \circ T+c$ acting as a representative of the class of all potentials defining the same Gibbs measure.

The goal of the present article is to study the geometry of the set of normalized potentials $\mathcal{N}$, of the normalization map $A \mapsto B$, and of the Gibbs map $A \mapsto \mu_{A}$. We give an easy proof of the fact that $\mathcal{N}$ is an analytic submanifold of $\mathcal{X}$ and that the normalization map is analytic; we compute the derivative of the Gibbs map; last we endow $\mathcal{N}$ with a natural weak Riemannian metric (derived from the asymptotic variance) with respect to which we compute the gradient flow induced by the pressure with respect to a given potential, e.g. the metric entropy functional. We also apply these ideas to recover in a wide setting existence and uniqueness of equilibrium states, possibly under constraints.

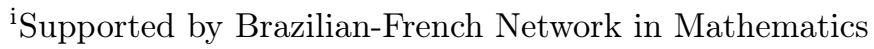

iiSupported by the Agence Nationale de la Recherche, grant ANR-11-JS01-0011.

iii Supported by CNPq-Brazil and INCTMat.

${ }^{\text {iv }}$ Partially supported by CNPq-Brazil through the postdoctoral scholarship PDJ/501839/2013-5.

2010 Mathematics Subject Classification: 37D35, 37A35, 37C30, 49Q20

Keywords: Transfer operators, Equilibrium states, Entropy, Regularity, Wasserstein space
} 


\section{Introduction}

The goal of this article is to propose a differential-geometric approach to the thermodynamical formalism for maps whose transfer operators satisfy the conclusion of the Ruelle-Perron-Frobenius theorem (for example, expanding maps).

Some of our results stated below are already known for certain dynamical systems (see later for more precise historical references); let us stress what we believe are our main contributions:

- we propose a point of view, based on differential geometry in the space of potentials, which provides new and efficient ${ }^{1}$ proofs of strong results (e.g. Fréchet dérivatives are computed instead of mere directional derivatives) valid in a fairly general framework,

- we compute an explicit formula for the derivative of $\int \varphi \mathrm{d} \mu_{A}$ with respect to $A$ (Theorem $\mathrm{C}$ ), leading naturally to the variance metric linking together several natural quantities (Theorem D),

- we use this metric to define the gradient of natural functionals, which leads to a gradient flow modeling a system out of equilibrium (Section 7.3.2),

- we show that the map sending a potential to its Gibbs measure is very far from being smooth in the sense of optimal transportation (Theorem E),

- we improve a result of Kucherenko and Wolf, identifying precisely the equilibrium state of a potential under a finite set of linear constraints (Theorem G, see also Example $\mathrm{H})$.

\subsection{Transfer operator, Gibbs measures and normalization}

Let $\Omega$ be a compact metric space and $T: \Omega \rightarrow \Omega$ be a finite-to-one map, defining a discrete-time dynamical system. The model cases are uniformly expanding maps such as $x \mapsto d x \bmod 1$ on the circle or the shift over right-infinite words on a finite alphabet, but we shall consider a very general setting by mostly asking ${ }^{2}$ that for each potential $A$ in a suitable function space $\mathcal{X}(\Omega)$, the Ruelle-Perron-Frobenius theorem holds for the transfer operator

$$
\begin{aligned}
\mathscr{L}_{A} & : \mathcal{X}(\Omega) \rightarrow \mathcal{X}(\Omega) \\
f & : x \mapsto \sum_{T(y)=x} e^{A(y)} f(y)
\end{aligned}
$$

i.e. $\mathscr{L}_{A}$ has a positive, simple leading eigenvalue $\lambda_{A}$ associated with a positive eigenfunction $h_{A}$; its dual operator acting on measures $\mathscr{L}_{A}^{*}$ has a unique eigenprobability

\footnotetext{
${ }^{1}$ See for example Corollary 3.6.

${ }^{2}$ see Section 2 for the precise hypotheses
} 
$\nu_{A}$; and $\mathscr{L}_{A}$ has a spectral gap below $\lambda_{A}$. Then, the measure $\mu_{A}=h_{A} \nu_{A}$ (where the multiplicative constant in $h_{A}$ is chosen so as to make $\mu_{A}$ a probability measure) is an invariant measure for $T$, which we will call here the Gibbs measure associated to the potential $A$.

Two different potentials $A, B$ which differ by a constant and a coboundary define the same Gibbs measure, which can thus be parametrized by the quotient space $\mathcal{Q}=\mathcal{X}(\Omega) / \mathcal{C}$ where

$$
\mathcal{C}=\{c+g-g \circ T \mid c \in \mathbb{R}, g \in \mathcal{X}(\Omega)\} .
$$

The subset $\mathcal{N} \subset \mathcal{X}(\Omega)$ of normalized potential (i.e. such that $\lambda_{A}=1$ and $h_{A}=\mathbf{1}$ ) contains exactly one representative of each class modulo $\mathcal{C}$, making $\mathcal{N}$ another natural parameter space for Gibbs measures.

Our main object of study is the first-order variation of $\mu_{A}$ with respect to $A$, which means we consider $\mu_{A+\zeta}$ for small $\zeta \in \mathcal{X}(\Omega)$; of course, adding to $\zeta$ a constant and a coboundary will have no effect. In the literature, it is often the case that one asks $\zeta$ to satisfy the normalizing condition $\int \zeta \mathrm{d} \mu_{A}=0$ to get rid of the constant, and then considers $\zeta$ up to coboundaries. We argue that instead, it makes things simpler and clearer to go fully with one of these points of view: either consider both $A$ and $\zeta$ modulo $\mathcal{C}$, or restrict to normalized $A$ and constrain $\zeta$ to be tangent to $\mathcal{N}$. Our fist result gives a solid ground to this principle (Theorem 3.4):

Theorem A. The set $\mathcal{N}$ of normalized potentials is an analytic submanifold of $\mathcal{X}(\Omega)$ and its tangent space at $A$ is $\operatorname{ker} \mathscr{L}_{A}$, which is a topological complement to $\mathcal{C}$.

From this we will easily deduce the analyticity and derivative of several important maps. Consider:

- the normalization map $N: \mathcal{X}(\Omega) \rightarrow \mathcal{N}$ which sends $A$ to the unique normalized potential in its class modulo $\mathcal{C}$,

- the leading eigenvalue map $\Lambda: A \mapsto \lambda_{A}$,

- the leading eigenfunction map $H: A \mapsto h_{A}$,

- the Gibbs map $G: A \mapsto \mu_{A}$ taking its values in $\mathcal{X}(\Omega)^{*}$ with the convention that $\mu_{A}(\varphi)=\int \varphi \mathrm{d} \mu_{A}$.

Then we get (Theorem 3.5 to Proposition 3.7):

Corollary B. The maps $N, \Lambda, H, G$ are analytic and for all $A \in \mathcal{X}(\Omega)$ :

- the differential $D N_{A}$ of $N$ at $A$ is the linear projection on $\operatorname{ker} \mathscr{L}_{N(A)}$ along $\mathcal{C}$,

- $D(\log \Lambda)_{A}=\mu_{A}$ as a linear form on $\mathcal{X}(\Omega)$, i.e.

$$
\left.\frac{\mathrm{d}}{\mathrm{d} t} \log \lambda_{A+t \zeta}\right|_{t=0}=\int \zeta \mathrm{d} \mu_{A} \quad \forall A, \zeta \in \mathcal{X}(\Omega) .
$$


The analyticity of these maps and the derivative of $\log \Lambda$ are well-known for many dynamical systems, ${ }^{3}$ however our framework is quite general (e.g. we do not assume any high-temperature hypothesis) and our method pretty elementary: we only use basic differential calculus, not complex analysis nor Kato's theory of regularity of eigendata for operators (as done, for example, in [PP90], [dSdSS14]).

\subsection{From integral differentiation to a Riemannian metric}

Both derivatives above are really easy to obtain, but the derivative of $G$ is slightly more complicated (Theorem 4.1):

Theorem C. For all $A, \varphi, \zeta \in \mathcal{X}(\Omega)$ we have

$$
\left.\frac{\mathrm{d}}{\mathrm{d} t} \int \varphi \mathrm{d} \mu_{A+t \zeta}\right|_{t=0}=\int\left(I-\mathscr{L}_{N(A)}\right)^{-1}\left(\varphi_{A}\right) \cdot D N_{A}(\zeta) \mathrm{d} \mu_{A}
$$

where $\varphi_{A}=\varphi-\int \varphi \mathrm{d} \mu_{A}$.

(Note that of course the left-hand-side is $D G_{A}(\zeta) \in \mathcal{X}(\Omega)^{*}$ applied at $\varphi$.)

This derivative can then be expressed in various forms using standard computations, see Sections 4 and 5, and some interesting connections appear.

Theorem D. All the following expressions

$$
\begin{aligned}
\langle\zeta, \eta\rangle_{A} & =D^{2}(\log \Lambda)_{A}(\zeta, \eta) \\
\langle\zeta, \eta\rangle_{A} & =\left.\frac{\mathrm{d}}{\mathrm{d} t} \int \eta \mathrm{d} \mu_{A+t \zeta}\right|_{t=0} \\
\langle\zeta, \zeta\rangle_{A} & =\operatorname{Var}\left(\zeta_{A}, \mu_{A}\right):=\lim _{n \rightarrow \infty} \frac{1}{n} \int\left(\sum_{i=0}^{n-1} \zeta_{A} \circ T^{i}\right)^{2} \mathrm{~d} \mu_{A} \\
\langle\zeta, \eta\rangle_{A} & =\int \zeta \eta \mathrm{d} \mu_{A} \quad \text { whenever } A \in \mathcal{N}, \zeta, \eta \in T_{A} \mathcal{N} .
\end{aligned}
$$

define the same analytic map $A \mapsto\langle\cdot, \cdot\rangle_{A}$ from $\mathcal{X}(\Omega)$ to the Banach space of symmetric linear 2-forms, such that $\langle\cdot, \cdot\rangle_{A}$ is positive-semi-definite with kernel equal to $\mathcal{C}$ for all $A$. This map induces by restriction a weak Riemannian metric on $\mathcal{N}$, and then by projection it induces a weak Riemannian metric on $\mathcal{Q}=\mathcal{X}(\Omega) / \mathcal{C}$.

The metric $\langle\cdot, \cdot\rangle_{A}$ is thus a close cousin to McMullen's variance metric introduced in the context of Teichmüller space $[\mathrm{McM} 08]^{4}$ (up a conformal rescaling by entropy), contains the derivative of the Gibbs map, controls the convexity of $\log \Lambda$ and extends the $L^{2}\left(\mu_{A}\right)$ metric on $\mathcal{N}$ at the same time.

\footnotetext{
${ }^{3}$ For an historical account of the problem, see the introduction of [BCV12] and references therein (among others [PP90],[Mañ90],[BS01]).

${ }^{4}$ See also [BCS15] by Bridgeman, Canary and Sambarino and references therein, and [PS14] by Pollicott and Sharp for an analogous metric of Weyl-Patterson type on spaces of metric graphs.
} 
In the closing Section 8, we show a concrete example of this approach. When the dynamics is just the shift on the Bernoulli space $\{1,2\}^{\mathbb{N}}$ and the potential depends only on two coordinates, we exhibit the metric explicitly and we compute the curvature (Proposition 8.1), which is positive. In analogy with the work of McMulleen, one could conjecture that when our metric is rescaled by the entropy, the curvature is strictly negative, but we show that this is not the case.

\subsection{The optimal transportation approach to the differentiability of measure-valued maps}

Above we took a very common point of view, considering the Gibbs map $G: A \mapsto \mu_{A}$ as taking value in (an affine subspace of) the Banach space $\mathcal{X}(\Omega)^{*}$, yielding an obvious differential structure in which each $\varphi \in \mathcal{X}(\Omega)$ defines a "coordinate function" by $\mu_{A} \mapsto$ $\mu_{A}(\varphi)=\int \varphi \mathrm{d} \mu_{A}$. We call this the "affine differential structure".

However, this is not the only way to study the regularity of such a map, and in Section 6 we study the "Wasserstein differential structure" aspect of the question. One can see $G$ as taking values in the subset $\mathcal{P}_{T}(\Omega)$ of $T$-invariant measures in the set $\mathcal{P}(\Omega)$ of all probability measures, and use the differential framework based on the 2-Wasserstein distance $W_{2}$ from optimal transportation which has been developed in the last fifteen years. ${ }^{5}$ This point of view proved useful in the study of the action of expanding circle maps near the absolutely continuous invariant measure by one of the present authors (see [Klo13, Klo15a]); here, we show that with the 2-Wasserstein metric the Gibbs map $A \mapsto \mu_{A}$ is far from being differentiable even in the simplest smoothest case.

Theorem E. Assume $T=x \mapsto d x \bmod 1$ is the standard $d$-self-covering map of the circle $\mathbb{S}^{1}=\mathbb{R} / \mathbb{Z}$ and $\mathcal{X}(\Omega)$ is the space of $\alpha$-Hölder functions for some $\alpha \in(0,1]$. Then given any smooth path $\left(A_{t}\right)$ in $\mathcal{X}(\Omega)$, the path of Gibbs measures $\left(\mu_{A_{t}}\right)$ is not absolutely continuous in $\left(\mathcal{P}(\Omega), W_{2}\right)$ unless it is constant.

Recall that a path in a metric space defined on an interval $I$ is said to be absolutely continuous when it has a metric speed in $L^{1}(I)$ : this is a very weak regularity, so that Theorem E can be interpreted as meaning that a small perturbation of the potential induces a brutal reallocation of the mass distribution in the sense of $W_{2}$. This contrasts with a Lipschitz regularity result obtained for the 1-Wasserstein metric in [KLS14] (but note that $W_{1}$ does not yield a differential structure).

\subsection{Applications to equilibrium states}

We end this introduction by presenting some applications and illustrations of our differential calculus setting and the metric obtained above.

\footnotetext{
${ }^{5}$ The full story does not fit in the bottom margin, but let us mention the important cornerstones which are the works of Otto [Ott01], Benamou and Brenier [BB00], and Ambrosio, Gigli and Savaré [AGS05]; see also [Vil03], [Vil09] and [Gig11].
} 
First we show that it is a nice framework to derive and extend to our framework the existence and uniqueness of equilibrium states. Consider the following optimization problems and induced functionals:

$$
\begin{aligned}
\mathrm{h}_{\mathcal{X}}(\mu) & :=\inf _{A \in \mathcal{X}(\Omega)} \log \lambda_{A}-\int A \mathrm{~d} \mu & & \text { for } \mu \in \mathcal{P}_{T}(\Omega) \\
\operatorname{Pr}(B) & :=\sup _{\mu \in \mathcal{P}_{T}(\Omega)} \mathrm{h}_{\mathcal{X}}(\mu)+\int B \mathrm{~d} \mu & & \text { for } B \in \mathcal{X}(\Omega)
\end{aligned}
$$

(recall that $\mathcal{X}(\Omega)$ is a suitable space of potentials $\Omega \rightarrow \mathbb{R}$ which we can chose with some freedom).

Theorem F. For all $B \in \mathcal{X}(\Omega)$, the supremum in the definition of $\operatorname{Pr}(B)$ is uniquely realized by $\mu_{B}$ and it holds $\operatorname{Pr}(B)=\log \lambda_{B}$.

We show in Remark 7.5 that for the case of the Classical Thermodynamical Formalism in the sense of [PP90] (the shift acting on the Bernoulli space) and for any invariant probability $\mu$ we have equality between $\mathrm{h}_{\mathcal{X}}(\mu)$ and the metric entropy of $\mu$. In this case the pressure $\operatorname{Pr}$ defined above also coincides with the usual topological pressure. We consider however more general hypothesis in our reasoning. We will refer to $h_{\mathcal{X}}$ and $\mathrm{Pr}$ as "entropy" and "pressure" from now on.

We then observe that the metric $\langle\cdot, \cdot\rangle_{A}$ enables us to define the gradient of various natural dynamical quantities, including entropy and pressure (see Proposition 7.10). This gives sense to the gradient flow of the functional

$$
A \mapsto \mathrm{h}_{\mathcal{X}}\left(\mu_{A}\right)+\int B \mathrm{~d} \mu_{A}
$$

obtained by composing $G$ with the functional defining the pressure. This gradient flow has a linear form when expressed in the quotient space $\mathcal{Q}$ and can serve as a model for non-equilibrium dynamics, according to which a system out of equilibrium behaves just like a system at equilibrium with a varying potential (Section 7.3.2). In case of a mere change in the temperature of the system's environment, this model predicts the physically sound property that the systems evolves only in its temperature (Remark 7.11).

As a consequence of Theorems D and $\mathrm{F}$ we obtain several results related to works by Kucherenko and Wolf. The first result, obtained in [KW14] under somewhat different hypotheses, is a prescription result. Given $\Phi=\left(\varphi_{1}, \ldots, \varphi_{K}\right)$ a tuple of test functions in $\mathcal{X}(\Omega)$, the "rotation vector"

$$
\operatorname{rv}(\mu)=\left(\int \varphi_{1}, \mathrm{~d} \mu, \ldots, \int \varphi_{K} \mathrm{~d} \mu\right)
$$

of a $T$-invariant measure describes some convex set $\operatorname{Rot}(\Phi) \subset \mathbb{R}^{K}$. The result is then that for all base potential $B$, every interior value of $\operatorname{Rot}(\Phi)$ can be realized uniquely as the Gibbs measure of a potential of the form $B+a_{1} \varphi_{1}+\cdots+a_{K} \varphi_{K}$ (Theorem 7.13). 
The second result states existence and uniqueness of equilibrium states under linear constraints; it is very close to Theorem B of [KW13], but even disregarding the difference in our hypotheses we obtain a more precise description of the equilibrium state: the parameter $s$ of [KW13] is always equal to 1 . In other words:

Theorem G. Let $\Phi=\left(\varphi_{1}, \ldots, \varphi_{K}\right) \in \mathcal{X}(\Omega)$ be such that $0 \in \operatorname{int} \operatorname{Rot}(\Phi)$. Given any $B \in \mathcal{X}(\Omega)$, the restriction of

$$
P_{B}: \mu \mapsto \mathrm{h}_{\mathcal{X}}(\mu)+\int B \mathrm{~d} \mu
$$

to the set $\mathcal{P}_{T}[\Phi]$ of invariant measures realizing $\int \varphi_{k} \mathrm{~d} \mu=0$ for all $k$ is uniquely maximized at the unique Gibbs measure in $\mathcal{P}_{T}[\Phi]$ that is defined by a potential of the form $B+a_{1} \varphi_{1}+\cdots+a_{K} \varphi_{K}$.

We also recover Theorem B in [KW14] (the supremum of entropy of measures realizing a given vector in the interior of $\operatorname{Rot}(\Phi)$ depends analytically on the vector, Corollary 7.16), and by nature our method could be applied to more general constraints (e.g. asking that $\operatorname{rv}(\mu)$ belongs to some submanifold of $\operatorname{Rot}(\Phi))$.

Theorem G notably shows that when $T$ is the shift map and the test functions and the potential $B$ all depend only on $n$ coordinates, so does the potential of the constrained equilibrium state, which is thus a $(n-1)$-Markovian measure (Remark 7.17 , which also follows from the results of [KW13] but is not stated there).

This result is precise enough to yield explicit solutions to some concrete maximizing questions, which as far as we know would be difficult to solve without it. Let us give a toy example which turns out to have a nontrivial answer.

Example H. Assume $T$ is the shift map on $\Omega=\{0,1\}^{\mathbb{N}}$. Among shift-invariant measures $\mu$ such that $\mu(01 *)=2 \mu(11 *)$, the Markov measure associated to the transition probabilities

$$
\begin{array}{ll}
\mathbb{P}(0 \rightarrow 0)=1-a & \mathbb{P}(0 \rightarrow 1)=a \\
\mathbb{P}(1 \rightarrow 0)=\frac{2}{3} & \mathbb{P}(1 \rightarrow 1)=\frac{1}{3}
\end{array}
$$

where $a$ is the only real solution to

$$
(1-a)^{5}=\frac{4}{27} a^{2} \quad(a \simeq 0.487803)
$$

uniquely maximizes entropy.

As a final remark, we mention that optimization problems such as we solve in Theorem $\mathrm{G}$ appear naturally in multifractal analysis, see [BS01], [BSS02], [Cli14]. Our approach might lead to explicit computation in that field. 


\section{Notation and preliminaries}

We shall consider the thermodynamical formalism associated with a discrete-time, continuous-space dynamical system. The phase space shall be denoted by $\Omega$, and will be assumed to be a compact metric space, whose metric shall be denoted by $d$. The time evolution is then described by a map $T: \Omega \rightarrow \Omega$ which will be assumed to be a finite-toone map. We will denote by $\mathcal{P}(\Omega)$ the set of probability measures on $\Omega$, and by $\mathcal{P}_{T}(\Omega)$ the subset of $T$-invariant measures.

Typical cases to serve as examples are the shift on $\mathcal{A}^{\mathbb{N}}$ where $\mathcal{A}$ is a finite alphabet, the maps $x \mapsto d x \bmod 1$ acting on the circle $\mathbb{S}^{1}=\mathbb{R} / \mathbb{Z}$. The reader not willing to deal with the detailed hypotheses below can assume $T$ is one of these classical maps.

Remark 2.1. We also want to consider cases such as the tent map

$$
x \mapsto \begin{cases}2 x & \text { if } x \leq \frac{1}{2} \\ 2-2 x & \text { if } x \geq \frac{1}{2}\end{cases}
$$

on the interval $[0,1]$. This map has a particularity shared with other map of the same kind: one point, $1 / 2$, has only one inverse image while its neighboring points have two. This will make it necessary to adjust some of the definitions below. Let us formalize a property of the tent map which we will refer to when we explain these modifications: the tent map has local inverse branches in the sense that for all $x \in \Omega$ there is an integer $d \geq 2$ (to be implicitly taken minimal), a neighborhood $V$ of $x$ and continuous maps $y_{k}: V_{k} \subset \Omega \rightarrow V$ (where $k \in\{1, \ldots, d\}$ ) such that for all $x^{\prime} \in V$ we have

$$
T^{-1}\left(x^{\prime}\right)=\left\{y_{1}\left(x^{\prime}\right), \ldots, y_{d}\left(x^{\prime}\right)\right\} .
$$

\subsection{Working Hypotheses}

\subsubsection{Space of potentials}

The first set of assumptions we make concerns the regularity of potentials; in designing the hypotheses below we tried to keep them general enough not to rule out non continuous potentials; e.g. in some settings bounded variation functions are meaningful (in particular when $T$ is only piecewise continuous).

We fix for all the article a space of functions $\mathcal{X}(\Omega)$, endowed with a norm $\|\cdot\|$, satisfying the following. 
(H1) $\mathcal{X}(\Omega)$ is a Banach space of Borel-measurable, bounded functions $\Omega \rightarrow \mathbb{R}$, which includes all constant functions; for all $f, g \in \mathcal{X}(\Omega)$ we have

$$
\|f g\| \leq\|f\|\|g\|
$$

for all $f \in \mathcal{X}(\Omega)$ that is positive and bounded away from 0 , the function $\log f$ also lies in $\mathcal{X}(\Omega)$; and for some constant $C$ it holds

$$
\|f\| \geq C \sup _{x \in \Omega}|f(x)|
$$

In particular for each probability measure $\mu$ on $\Omega$, the linear form defined by $f \mapsto$ $\int f \mathrm{~d} \mu$ is continuous: in other words, every probability measure can be seen as an element of $\mathcal{X}(\Omega)^{*}$.

Note that when $f \in \mathcal{X}(\Omega)$ is positive and bounded away from $0,1 / f=e^{-\log f}$ is also in $\mathcal{X}(\Omega)$.

Remark 2.2. In some circumstances, one works with a norm satisfying only the weak multiplicativity condition $\|f g\| \leq C\|f\|\|g\|$ for some positive constant $C$. Then one can define a new, equivalent norm $\|\cdot\|^{\prime}=C\|\cdot\|$ which is then multiplicative.

Example 2.3. The space $\operatorname{Hol}_{\alpha}(\Omega)$ of $\alpha$-Hölder functions (for some $\alpha \in(0,1]$ ) with its usual norm

$$
\|f\|_{\alpha}=\sup _{x \in \Omega}|f(x)|+\sup _{x \neq y \in \Omega} \frac{|f(x)-f(y)|}{d(x, y)^{\alpha}}
$$

satisfies (H1). When $\alpha=1$, we get the space $\operatorname{Lip}(\Omega)$ of Lipschitz-continuous functions. Note that $d^{\alpha}$ is a distance on $\Omega$, and that $\operatorname{Hol}_{\alpha}(\Omega)$ coincide with $\operatorname{Lip}\left(\Omega, d^{\alpha}\right)$.

Next, we need a compatibility hypothesis between $T$ and $\mathcal{X}(\Omega)$.

(H2) $T$ preserves $\mathcal{X}(\Omega)$ forward and backward, i.e. the composition operator $f \mapsto f \circ T$ is well-defined and continuous from $\mathcal{X}(\Omega)$ to itself, and or all $f \in \mathcal{X}(\Omega)$, we have

$$
g: x \mapsto \sum_{T(y)=x} f(y) \in \mathcal{X}(\Omega) \quad \text { and } \quad\|g\| \leq C\|f\|
$$

for some constant $C$ (i.e. $f \mapsto g$ is a continuous operator on $\mathcal{X}(\Omega)$ ).

Example 2.4. When $\mathcal{X}(\Omega)=\operatorname{Hol}_{\alpha}(\Omega)$, it is sufficient to ask the map $T$ to be a local bi-Lipschitz homeomorphism to obtain (H2).

Remark 2.5. The tent map does not strictly speaking satisfy this compatibility when for example $\mathcal{X}(\Omega)=\operatorname{Hol}_{\alpha}(\Omega)$, because $\frac{1}{2}$ only has one inverse image and $g$ is usually not even continuous. One can fix such cases by introducing a suitable weight in all sums $\sum_{T(y)=x} f(y)$, i.e. $\sum_{T(y)=\frac{1}{2}} f(y)$ should be interpreted as $f(1)+f(1)$ to ensure continuity in $x$ of $\sum_{T(y)=x} f(y)$. In other words, if needed $\sum_{T(y)=x} f(y)$ can be replaced everywhere by $\sum_{k} f\left(y_{k}(x)\right)$ where $y_{k}$ are the local inverse branches of $T$. 


\subsubsection{Transfer operator}

The composition operator arising from $T$ is the natural functional counterpart to our dynamical system; in fact, most properties of ergodic flavor of $T$ are naturally formulated in terms of the composition operator on a certain class of functions. However it is useful to investigate its "inverses", the transfer operators. Given a "potential" $A \in \mathcal{X}(\Omega)$, one defines a transfer operator (also called a Ruelle operator) by

$$
\mathscr{L}_{A}(f)(x)=\sum_{T(y)=x} e^{A(y)} f(y)
$$

note that since $\mathcal{X}(\Omega)$ is a Banach algebra, $e^{A}$ lies in $\mathcal{X}(\Omega)$ and so does $\mathscr{L}_{A}(f)$; hypothesis (H2) also implies that $\mathscr{L}_{A}$ is a continuous operator.

Since $\mathcal{X}(\Omega)$ is a space of functions, it contains a canonical "positive cone", the set of positive functions, which is convex and invariant by dilation. By design, the transfer operator is positive in the sense that it maps the positive cone into itself. Typical expanding assumptions for $T$ ensure that the positive cone is even mapped into a narrower cone, inducing a contraction on the set of positive directions endowed with a suitable distance (see e.g. [Bal00]). Instead of assuming such kind of hypothesis on $T$, we shall only assume the consequences that are usually drawn from them. Namely, we ask that $(T, \mathcal{X}(\Omega)$ ) satisfies a Ruelle-Perron-Frobenius theorem (including a spectral gap) in the sense of the following two hypotheses.

(H3) For all $A \in \mathcal{X}(\Omega)$ the transfer operator $\mathscr{L}_{A}$ has a positive maximal eigenvalue $\lambda_{A}$ and a positive, bounded away from 0 eigenfunction $h_{A} \in \mathcal{X}(\Omega)$ :

$$
\mathscr{L}_{A}\left(h_{A}\right)=\lambda_{A} h_{A}
$$

and the dual operator $\mathscr{L}_{A}^{*}$ of $\mathscr{L}_{A}$ preserves the set of finite measures and has a eigenmeasure $\nu_{A} \in \mathcal{P}(\Omega)$ for the eigenvalue $\lambda_{A}$, in particular

$$
\int \mathscr{L}_{A}(f) \mathrm{d} \nu_{A}=\lambda_{A} \int f \mathrm{~d} \nu_{A} \quad \forall f \in \mathcal{X}(\Omega)
$$

Observe that when all functions of $\mathcal{X}(\Omega)$ are continuous, $\mathscr{L}_{A}$ extends to all continuous functions and then the dual operator automatically acts on measures.

(H4) For all $A \in \mathcal{X}(\Omega)$, there are positive constants $D, \delta$ such that for all $n \in \mathbb{N}$ and all $f \in \mathcal{X}(\Omega)$ such that $\int f \mathrm{~d} \nu_{A}=0$, we have

$$
\left\|\mathscr{L}_{A}^{n}(f)\right\| \leq D \lambda_{A}^{n}(1-\delta)^{n}\|f\| .
$$

It follows in particular that $\lambda_{A}$ is a simple eigenvalue and that $\nu_{A}$ defines a natural (topological) complement to its eigendirection. 
It is easy to see that $\mu_{A}=h_{A} \nu_{A}$ defines an invariant measure for $T$, and up to normalizing $h_{A}$ we can assume $\mu_{A}$ is a probability measure which we will call the Gibbs measure of $A$.

Example 2.6. When $T$ is expanding in a relatively general sense and $\mathcal{X}(\Omega)$ is a space of Hölder functions, (H3) and (H4) are proved in [KLS14] (the spectral gap is proved there for normalized potentials only, but see remark 2.9).

\subsubsection{Further hypotheses}

Our first results will only use (H1) to (H4), but at some point we will need two further hypotheses, which feel harmless (in the sense that they hold for most if not all relevant examples), but which do not follow from the previous ones.

From Section 5 on, we will assume:

(H5) For all $A, f \in \mathcal{X}(\Omega)$, if $f$ is non-negative and $\int f \mathrm{~d} \mu_{A}=0$ then $f=0$.

Remark 2.7. If all functions in $\mathcal{X}(\Omega)$ are continuous, it is sufficient to ask that $\mu_{A}$ has full support for all $A$ to ensure (H5).

Example 2.8. Assume that $T$ is continuous, that all functions in $\mathcal{X}(\Omega)$ are continuous, and that the only closed subsets $A \subset \Omega$ which are both forward and backward invariant (i.e. $T(A)=T^{-1}(A)=A$ ) are the empty set $\varnothing$ and the full space $\Omega$. Then (H5) holds.

Indeed, since $\mu_{A}$ is an invariant measure, its support is a closed invariant subset of $\Omega$. But (assuming without lost of generality that $A$ is normalized, see below) the invariance under $\mathscr{L}_{A}^{*}$ and the fact that $e^{A}$ is a positive function also implies that $\operatorname{supp} \mu_{A}$ is backward invariant, so that $\mu_{A}$ must have full support. The continuity of $f$ then gives the conclusion.

In Section 7 we will use the following largeness hypothesis, meant to avoid degenerate cases such as $\mathcal{X}(\Omega)=\{$ constants $\}$.

(H6) All continuous functions $f: \Omega \rightarrow \mathbb{R}$ can be uniformly approximated by elements of $\mathcal{X}(\Omega)$.

(Note that we do not imply here that the functions in $\mathcal{X}(\Omega)$ are continuous themselves.)

\subsection{Normalization}

Among the potentials, of particular importance are the normalized ones, i.e. those potentials $A$ such that $\mathscr{L}_{A}(\mathbf{1})=\mathbf{1}$ (where $\mathbf{1}$ denotes the constant function with value 1 ) i.e. such that $\lambda_{A}=1$ and $h_{A}=1$. In other words, $A$ is normalized when

$$
\sum_{T(y)=x} e^{A}(y)=1 \quad \forall x \in \Omega .
$$

Two nice properties that give a first evidence for the relevance this definition are that when $A$ is normalized, first $\mathscr{L}_{A}$ is a left-inverse to the composition operator:

$$
\mathscr{L}_{A}(f \circ T)=f \quad \forall f \in \mathcal{X}(\Omega),
$$


second $\mathscr{L}_{A}^{*}$ preserves the set of probability measures. One can then interpret $\mathscr{L}_{A}^{*}$ as a Markov chain, the numbers $e^{A(y)}$ representing the probability of transiting from $x$ to $y$ whenever $T(y)=x$; a realization of this Markov chain is a random reverse orbit of $T$.

As is well-known, the Ruelle-Perron-Frobenius theorem enables one to "normalize" a potential $A$, by writing

$$
B=A+\log h_{A}-\log h_{A} \circ T-\log \lambda_{A} \in \mathcal{X}(\Omega) .
$$

Then one gets

$$
\begin{aligned}
& \mathscr{L}_{B}(f): x \mapsto \sum_{T(y)=x} e^{A(y)} \frac{h_{A}(y)}{\lambda_{A} h_{A}(x)} f(y) \\
& \mathscr{L}_{B}(f)=\frac{1}{\lambda_{A} h_{A}} \mathscr{L}_{A}\left(h_{A} f\right)
\end{aligned}
$$

where (H3) ensures that $h_{A}$ is bounded away from 0 and (H1) then ensures that $1 / h_{A} \in$ $\mathcal{X}(\Omega)$. The transfer operators $\mathscr{L}_{A}$ and $\mathscr{L}_{B}$ are thus conjugated one to another up to a multiplicative constant $\lambda_{A}$, the conjugating operator being the multiplication by $h_{A}$; in particular

$$
\mathscr{L}_{B}(\mathbf{1})=\frac{1}{\lambda_{A} h_{A}} \mathscr{L}_{A}\left(h_{A}\right)=\mathbf{1}
$$

This conjugacy shows that the Gibbs measure $\mu_{A}=h_{A} \nu_{A}$ is also the eigenprobability $\nu_{B}=\mu_{B}$ of $\mathscr{L}_{B}^{*}$; each potential yields an invariant probability measure, but several potentials can yield the same Gibbs measure.

Using the same computation than above, one sees that whenever two arbitrary potentials $A, B$ are related as above, i.e. $B=A+g-g \circ T+c$ for some $g \in \mathcal{X}(\Omega)$ and $c \in \mathbb{R}$, then their transfer operators and their duals are conjugated one to another up to a constant:

$$
\mathscr{L}_{B}(\cdot)=e^{c} e^{-g} \mathscr{L}_{A}\left(e^{g} \cdot\right) \quad \mathscr{L}_{B}^{*}(\cdot)=e^{c} e^{g} \mathscr{L}_{A}^{*}\left(e^{-g} \cdot\right)
$$

where $e^{g}$ is in $\mathcal{X}(\Omega)$, positive and bounded away from 0 . It follows immediately that (up to normalizing constants) $h_{B}=h_{A} e^{-g}, \nu_{B}=e^{g} \nu_{A}$ and $\lambda_{B}=e^{c} \lambda_{A}$. In particular we have $\mu_{B}=\mu_{A}$ : both potentials define the same Gibbs measure. It is also straightforward to check that if moreover both $A$ and $B$ are normalized, $g$ must be a constant and $c$ must be zero, so that $A=B$. In other words, we have a subspace

$$
\mathcal{C}=\{g-g \circ T+c \mid g \in \mathcal{X}(\Omega), c \text { a constant }\} \subset \mathcal{X}(\Omega)
$$

such that each class modulo $\mathcal{C}$ defines one Gibbs measure, and contains exactly one normalized potential. One says that a function of the form $g-g \circ T$ is a coboundary, thus $\mathcal{C}$ is the space generated by coboundaries and constants. All the above is very classical; our goal is now to study in more details the following objects:

- the set $\mathcal{N} \subset \mathcal{X}(\Omega)$ of normalized potentials (which is not a linear subspace, see Remark 5.4), 
- the normalization map

$$
N: A \mapsto A+\log h_{A}-\log h_{A} \circ T-\log \lambda_{A}
$$

from $\mathcal{X}(\Omega)$ to $\mathcal{N}$

- the quotient $\mathcal{Q}=\mathcal{X}(\Omega) / \mathcal{C}$, and

- the Gibbs map $G: A \mapsto \mu_{A}$ from $\mathcal{X}(\Omega)$, seen as taking value either in $\mathcal{X}(\Omega)^{*}$ or in $\mathcal{P}_{T}(\Omega) \subset \mathcal{P}(\Omega)$

The typical questions we want to answer are of differential-geometric flavor: is $\mathcal{N}$ a submanifold of $\mathcal{X}(\Omega)$ ? Are the maps $N$ and $G$ differentiable? How to endow $\mathcal{N}$ or $\mathcal{Q}$ with a meaningful Riemannian metric? Can we then study gradient flows of natural functionals on these spaces?

Remark 2.9. The conjugacy between the transfer operator of a potential $A$ and the transfer operator of its normalization $B=N(A)$ shows that a spectral gap for $\mathscr{L}_{B}$ implies the same spectral gap for $\mathscr{L}_{A}$ (with a different constant $D$, but the same $\delta$ ). Indeed, if $\int f \mathrm{~d} \nu_{A}=0$ then $\int f / h_{A} \mathrm{~d} \mu_{A}=0$ and

$$
\begin{aligned}
\left\|\mathscr{L}_{A}^{n}(f)\right\| & =\left\|\lambda_{A}^{n} h_{A} \mathscr{L}_{B}^{n}\left(f / h_{A}\right)\right\| \\
& \leq \lambda_{A}^{n}\left\|h_{A}\right\| D(1-\delta)^{n}\left\|f / h_{A}\right\| \\
& \leq\left(D\left\|h_{A}\right\|\left\|1 / h_{A}\right\|\right) \lambda_{A}^{n}(1-\delta)^{n}\|f\| .
\end{aligned}
$$

In particular, if hypothesis (H3) is satisfied, the spectral gap for normalized potentials implies (H4) for all potentials.

Remark 2.10. The spectral gap hypothesis implies an exponential decay of correlation for functions in $\mathcal{X}(\Omega)$ : indeed if $A$ is any potential and $f, g \in \mathcal{X}(\Omega)$ are such that $\int f \mathrm{~d} \mu_{A}=0$, we have

$$
\begin{aligned}
\left|\int f \cdot g \circ T^{n} \mathrm{~d} \mu_{A}\right| & =\left|\int \mathscr{L}_{N(A)}^{n}\left(f \cdot g \circ T^{n}\right) \mathrm{d} \mu_{A}\right| \\
& =\left|\int \mathscr{L}_{N(A)}^{n}(f) \cdot g \mathrm{~d} \mu_{A}\right| \\
& \leq\left\|\mathscr{L}_{N(A)}^{n}(f) \cdot g\right\|_{\infty} \\
& \leq C^{-2}\left\|\mathscr{L}_{N(A)}^{n}(f)\right\|\|g\| \\
& \leq C^{-2} D(1-\delta)^{n}\|f\|\|g\|
\end{aligned}
$$

Remark 2.11. In typical situations, a normalized potential $A$ can be recovered from the Gibbs measure as a Jacobian: for example, if $T$ has inverse branches $y_{i}$ near each $x \in \Omega$ which are local homeomorphisms with disjoint images, then

$$
e^{A\left(y_{i}(x)\right)}=\frac{\mathrm{d} \mu_{A}\left(y_{i}(x)\right)}{\mathrm{d} \mu_{A}(x)}
$$


in the sense that if $B=B(x, \varepsilon)$ is a small ball around $x$, the ratio of $\mu_{A}\left(y_{i}(B)\right)$ with respect to $\mu_{A}(B)$ goes to $e^{A\left(y_{i}(x)\right)}$ when $\varepsilon$ goes to zero (in doubt, integrate caracteristic functions of balls with respect to one measure and change variables to verify such claim). Slight adaptations of this argument are needed for example for tent maps.

In general, it might a priori happen that two different normalized potentials $A, A^{\prime}$ have the same Gibbs measure. We will see much later in Remark 7.4 that our assumptions are sufficient to prevent this, and ensure perfect identifications between normalized potentials, $\bmod \mathcal{C}$ classes of potentials, and Gibbs measures.

\subsection{Analytic maps and submanifolds}

When working in infinite-dimensional spaces, just as differentiability has various definitions of varying strength (Gâteaux versus Fréchet), the analyticity of a map can be defined in several ways. Here, we take the strongest definition, recalled below.

First, recall that a closed linear subspace $M$ in a Banach space $\mathcal{X}$ is said to be topologically complemented, or for short complemented, when there is a closed linear subspace $N$ which is an algebraic complement. We shall only write $\mathcal{X}=M \oplus N$ when $M$ and $N$ are topological complements. The projection to $M$ along $N$ and the projection to $N$ along $M$ are then continuous, i.e. for all $x \in \mathcal{X}$, the decomposition $x=m+n$ with $m \in M$ and $n \in N$ exists, is unique, and $m$ and $n$ depend linearly continuously on $x$. Equivalently, $M$ is complemented when it is the image, or the kernel, of a continuous linear projection $\mathcal{X} \rightarrow \mathcal{X}$.

Let $\mathcal{X}$ and $\mathcal{Y}$ be two Banach spaces, whose norms will both be denoted by $\|\cdot\|$. A continuous, symmetric, multilinear operator $a: \mathcal{X}^{k} \rightarrow \mathcal{Y}$ has an operator norm denoted by $|a|$; if $\zeta$ is a vector in $\mathcal{X}$, we denote by $\zeta^{(k)}$ the element $(\zeta, \zeta, \ldots, \zeta)$ of $\mathcal{X}^{k}$ and we have

$$
\left\|a\left(\zeta^{(k)}\right)\right\| \leq|a|\|\zeta\|^{k} .
$$

We shall say that a sequence $a_{k}: \mathcal{X}^{k} \rightarrow \mathcal{Y}$ of such $k$-ary operators $(k \geq 0)$ is a series with positive radius of convergence if the complex series

$$
\sum_{k \geq 0}\left|a_{k}\right| z^{k}
$$

has a positive radius of convergence in $\mathbb{C}$.

Let $\Phi: U \subset \mathcal{X} \rightarrow \mathcal{Y}$ be a map defined from an open subset of $\mathcal{X}$. We say that $\Phi$ is analytic if for each $x \in U$ there is a series of $k$-linear, symmetric, continuous operators $a_{k}: \mathcal{X}^{k} \rightarrow \mathcal{Y}$ with positive radius of convergence such that on an open subset of $U$ the following identity holds:

$$
\Phi(x+\zeta)=\sum_{k \geq 0} a_{k}\left(\zeta^{(k)}\right) .
$$

An analytic map is smooth (in particular, Fréchet differentiable and locally Lipschitzcontinuous) and the operators $a_{k}$ are uniquely defined by $\Phi$. Most classical results hold in this context, in particular the inverse function theorem and the implicit function theorem (see [Cha85] and [Whi65]). 
More precisely, a map $\Phi: U \subset \mathcal{X} \rightarrow \mathcal{Y}$ which is analytic and such that $D \Phi_{x}: \mathcal{X} \rightarrow \mathcal{Y}$ is a topological isomorphism for each $x$, has a local reciprocal near each point, which is itself analytic (inverse function theorem); if a map $F: U \subset \mathcal{X} \rightarrow \mathcal{Y}$ is such that $F(x)=0$ for some $x \in U, D F_{x}$ is onto $\mathcal{Y}$ and ker $D F_{x}$ is complemented in $\mathcal{X}$, then the level set $F^{-1}(0)$ is an analytic submanifold of $\mathcal{X}$ in a neighborhood of $x$ (implicit function theorem). In particular, this means that there is an analytic diffeomorphism defined in a neighborhood of $x$ that maps $F^{-1}(0)$ to (an open set of) a complemented, closed, linear subspace of $\mathcal{X}$; it also means that $F^{-1}(0)$ can be locally written as the graph of an analytic map over a complemented subspace.

\section{Normalizing potentials}

We will now consider the set of normalized potentials

$$
\mathcal{N}=\left\{A \in \mathcal{X} \mid \mathscr{L}_{A}(\mathbf{1})=\mathbf{1}\right\}
$$

and the normalization map $N$ that sends any potential $A$ to its normalization:

$$
N(A)=A-\log \lambda_{A}+\log h_{A}-\log h_{A} \circ T .
$$

The map $N$ can be described as the (non-linear) projection on $\mathcal{N}$ along

$$
\mathcal{C}=\{g-g \circ T+c \mid g \in \mathcal{X}(\Omega), c \text { a constant }\} .
$$

We start by a simple Lemma which will both prove useful and serve as an example of the use of convergent series in our study. We shall denote by ker $\mu_{A} \subset \mathcal{X}(\Omega)$ the kernel of $\mu_{A}$ seen as a linear form, i.e.

$$
\operatorname{ker} \mu_{A}:=\left\{f \in \mathcal{X}(\Omega) \mid \int f \mathrm{~d} \mu_{A}=0\right\} .
$$

Lemma 3.1. If $A$ is a normalized potential, the operator $I-\mathscr{L}_{A}$ is onto ker $\mu_{A}$, and its corestriction to ker $\mu_{A}$ has a continuous inverse given by

$$
\left(I-\mathscr{L}_{A}\right)^{-1}=\sum_{k=0}^{\infty} \mathscr{L}_{A}^{k} \quad: \quad \operatorname{ker} \mu_{A} \rightarrow \mathcal{X}(\Omega) .
$$

Proof. For all $f \in \mathcal{X}(\Omega)$, we have that

$$
\int\left(I-\mathscr{L}_{A}\right)(f) \mathrm{d} \mu_{A}=\int f \mathrm{~d} \mu_{A}-\int f \mathrm{~d}\left(\mathscr{L}_{A}^{*} \mu_{A}\right)=0,
$$

because $\mu_{A}$ is fixed by $\mathscr{L}_{A}^{*}$. It follows that $I-\mathscr{L}_{A}$ takes its values in ker $\mu_{A}$.

For all $f \in \operatorname{ker} \mu_{A}$ and all $n$, we have

$$
\left(I-\mathscr{L}_{A}\right)\left(\sum_{k=0}^{n} \mathscr{L}_{A}^{k}(f)\right)=f-\mathscr{L}_{A}^{n+1}(f) .
$$


By the spectral gap assumption, we obtain that $\sum \mathscr{L}_{A}^{k}(f)$ converges, that it is bounded by $\frac{D}{\delta}\|f\|$, and that $\mathscr{L}_{A}^{n+1}(f)$ goes to 0 when $n \rightarrow \infty$. We deduce that $\sum_{k=0}^{\infty} \mathscr{L}_{A}^{k}$ is well-defined and a right-inverse to $I-\mathscr{L}_{A}$, which is therefore onto ker $\mu_{A}$.

By commutation the above shows that, for all $f \in \mathcal{X}(\Omega)$,

$$
\sum_{k=0}^{n} \mathscr{L}_{A}^{k}\left(\left(I-\mathscr{L}_{A}(f)\right)\right.
$$

converges to $f$, so that we have defined an inverse to (a corestriction of) $I-\mathscr{L}_{A}$.

This has useful consequences, which will be better phrased by introducing another operator related to $A$.

Definition 3.2. Given any normalized potential $A$, let $\mathscr{M}_{A}$ be the continuous linear operator on $\mathcal{X}(\Omega)$ defined by

$$
\mathscr{M}_{A}(f)=-\left(I-\mathscr{L}_{A}\right)^{-1} \circ \mathscr{L}_{A}\left(f_{A}\right)=-\sum_{k=1}^{\infty} \mathscr{L}_{A}^{k}\left(f_{A}\right),
$$

where $f_{A}:=f-\int f \mathrm{~d} \mu_{A}$.

Observe that $\mathscr{L}_{A}$ maps ker $\mu_{A}$ to itself, so that $\mathscr{M}_{A}$ is indeed well-defined and takes its values in ker $\mu_{A}$, and that $\mathscr{M}_{A}$ commutes with $\mathscr{L}_{A}$.

Proposition 3.3. Let $A$ be a normalized potential. Then:

1. given $f \in \mathcal{C}$, there is a unique decomposition $f=g-g \circ T+c$ with $g \in \operatorname{ker} \mu_{A}$ and $c$ a constant, given by

$$
c=\int f \mathrm{~d} \mu_{A} \quad \text { and } \quad g=\mathscr{M}_{A}(f),
$$

2. the subspace $\mathcal{C}$ is closed in $\mathcal{X}(\Omega)$, so that $\mathcal{Q}=\mathcal{X}(\Omega) / \mathcal{C}$ inherits a Banach space structure from $\|\cdot\|$,

3. $\operatorname{ker} \mathscr{L}_{A}$ and $\mathcal{C}$ are (topological) complements in $\mathcal{X}$,

4. $\mathscr{L}_{A}$ maps $\mathcal{C}$ onto $\mathcal{X}(\Omega)$.

Proof. First observe that given any decomposition $f=g-g \circ T+c$ and any $T$-invariant probability measure $\mu$, we have

$$
\int f \mathrm{~d} \mu=\int g \mathrm{~d} \mu-\int g \mathrm{~d}\left(T_{\#} \mu\right)+c=c
$$

where $T_{\#} \mu_{A}$ is the usual pushforward of the measure $\mu_{A}$ with respect to $T$. Since $\mu_{A}$ is invariant, it follows that $c$ must equal $\int f \mathrm{~d} \mu_{A}$ and is uniquely defined. 
Let us then check that any $f \in \mathcal{C} \cap \operatorname{ker} \mathscr{L}_{A}$ must vanish. First, it is easy to see that $\operatorname{ker} \mathscr{L}_{A} \subset \operatorname{ker} \mu_{A}$ :

$$
\int f \mathrm{~d} \mu_{A}=\int f \mathrm{~d}\left(\mathscr{L}_{A}^{*} \mu_{A}\right)=\int \mathscr{L}_{A}(f) \mathrm{d} \mu_{A}=0 .
$$

It follows that we can write $f=g-g \circ T$, so that

$$
0=\mathscr{L}_{A}(g-g \circ T)=\mathscr{L}_{A}(g)-g
$$

and $g$ is an eigenfunction of $\mathscr{L}_{A}$ for the eigenvalue 1 . Therefore $g$ is constant and $f=0$.

To prove 1 , we write $f=g_{1}-g_{1} \circ T+c$ for some $g_{1}$ and with $c=\int f \mathrm{~d} \mu_{A}$. Setting $g=g_{1}-\int g_{1} \mathrm{~d} \mu_{A}$, we still have $f=g-g \circ T+c$ and

$$
\mathscr{L}_{A}(f-c)=\mathscr{L}_{A}(g-g \circ T)=\mathscr{L}_{A}(g)-g
$$

since $\mathscr{L}_{A}$ is a left-inverse to the composition operator. Now, from $g \in$ ker $\mu_{A}$ it follows $g=-\left(I-\mathscr{L}_{A}\right)^{-1} \mathscr{L}_{A}(f-c)=\mathscr{M}_{A}(f)$, as claimed.

To prove 2 , consider a sequence of functions $f_{n} \in \mathcal{C}$ which converges to $f \in \mathcal{X}(\Omega)$. Then using 1 , we can write $f_{n}=g_{n}-g_{n} \circ T+c_{n}$ where $g_{n}, c_{n}$ are images of $f_{n}$ by continuous operators. In particular $g_{n}$ and $c_{n}$ have limits $g \in \mathcal{X}(\Omega)$ and $c \in \mathbb{R}$, so that $f=g-g \circ T+c \in \mathcal{C}$.

To prove 3 , since we already know that ker $\mathscr{L}_{A}$ and $\mathcal{C}$ intersect trivially, we consider any $f \in \mathcal{X}(\Omega)$ and let $c:=\int f \mathrm{~d} \mu_{A}$ and $g=\mathscr{M}_{A}(f)$. We have

$$
\mathscr{L}_{A}(g-g \circ T+c)=\mathscr{L}_{A}(g)-g+c \mathscr{L}_{A}(\mathbf{1})=\mathscr{L}_{A}(f-c)+c=\mathscr{L}_{A}(f)
$$

where the second equality follows from $\left(\mathscr{L}_{A}-I\right) \mathscr{M}_{A}=\mathscr{L}_{A}$ on ker $\mu_{A}$. It follows that $\ell:=f-(g-g \circ T+c)$ is an element of ker $\mathscr{L}_{A}$. The decomposition

$$
f=\ell+(g-g \circ T+c)
$$

shows that $\mathcal{X}(\Omega)=\operatorname{ker} \mathscr{L}_{A}+\mathcal{C}$ and since both spaces are closed, ker $\mathscr{L}_{A}$ and $\mathcal{C}$ are complements.

To prove 4 , let $f \in \mathcal{X}(\Omega)$ and set $c=\int f \mathrm{~d} \mu_{A}$ and $g:=\left(I-\mathscr{L}_{A}\right)^{-1}(c-f)$. Now $g-g \circ T+c$ is an element of $\mathcal{C}$, and we have

$$
\begin{aligned}
\mathscr{L}_{A}(g-g \circ T+c) & =\mathscr{L}_{A}(g)-g+c \\
& =-\left(I-\mathscr{L}_{A}\right)(g)+c \\
& =f-c+c=f .
\end{aligned}
$$

We are know in a position to prove our first main result, that $\mathcal{N}$ is an analytic submanifold of $\mathcal{X}(\Omega)$. This result might be known, but we did not find a clear statement in the literature, related statements are often framed into a weaker definition of analyticity, the identification of the tangent space seems new, and we obtain the result without resorting to complex analysis as usually used to prove the regularity of the eigendata of operators (see appendix V in [PP90], where the weak definition of analyticity should be noted, and also section 3.3 in [BS01]). We shall in fact deduce from Theorem 3.4 that the leading eigenvalue and positive eigenfunction of $\mathscr{L}_{A}$ both depend analytically on $A$. 
Theorem 3.4. The set $\mathcal{N}$ of normalized potentials is an analytic submanifold of $\mathcal{X}(\Omega)$, and its tangent space at $A \in \mathcal{N}$ is $T_{A} \mathcal{N}=\operatorname{ker} \mathscr{L}_{A}$.

Proof. This is a direct consequence of the implicit function theorem.

Let $F: \mathcal{X}(\Omega) \rightarrow \mathcal{X}(\Omega)$ be the map defined by

$$
F(A)(x)=\mathscr{L}_{A}(\mathbf{1})(x)=\sum_{T y=x} e^{A(y)} .
$$

Then $F$ is analytic, as follows from the analyticity of the exponential:

$$
F(A+\zeta)=\sum_{k \geq 0} D^{k} F_{A}(\zeta)
$$

where

$$
D^{k} F_{A}\left(\zeta_{1}, \ldots, \zeta_{k}\right)(x):=\sum_{T y=x} e^{A(y)} \frac{\prod_{i=1}^{k} \zeta_{i}(y)}{k !}
$$

defines a series of continuous, symmetric $k$-linear operators with infinite radius of convergence (note that we use here the assumptions that $\mathcal{X}(\Omega)$ has multiplicative norm, and that $\sum_{T y=x} \zeta(y)$ is in $\mathcal{X}(\Omega)$ for all $\zeta \in \mathcal{X}(\Omega)$ ).

Now, given a potential $A$ and a vector $\zeta$ both in $\mathcal{X}(\Omega)$, we have

$$
D F_{A}(\zeta)=\sum_{T y=x} e^{A(y)} \zeta(y)=\mathscr{L}_{A}(\zeta)
$$

so that $D F_{A}=\mathscr{L}_{A}$; since we know from Proposition 3.3 that ker $\mathscr{L}_{A}$ is complemented and $\mathscr{L}_{A}$ is onto $\mathcal{X}(\Omega)$, we can apply the implicit function theorem.

We also get directly the analyticity of the normalization map as explained in the last paragraph of Section 2.3

Theorem 3.5. The normalization map $N: \mathcal{X}(\Omega) \rightarrow \mathcal{N}$ sending a potential to its normalized version is analytic. Moreover, its derivative $D N_{A}$ at a point $A \in \mathcal{X}(\Omega)$ is the linear projection on $T_{N(A)} \mathcal{N}=\operatorname{ker} \mathscr{L}_{N(A)}$ in the direction of $\mathcal{C}$.

Proof. See figure 1 for a general picture of the various maps involved. Let $\Pi: \mathcal{X}(\Omega) \rightarrow \mathcal{Q}$ be the quotient map; it is a continuous linear map, and in particular it is analytic. Its restriction $\Pi_{\mid \mathcal{N}}$ to the submanifold $\mathcal{N}$ is therefore an analytic map, and we have for all $A \in \mathcal{N}:$

$$
D\left(\Pi_{\mid \mathcal{N}}\right)_{A}=\Pi_{\mid T_{A} \mathcal{N}}=\Pi_{\mid \operatorname{ker} \mathscr{L}_{A}} .
$$

Since ker $\mathscr{L}_{A}$ and $\mathcal{C}$ are topological complements, this differential is invertible with continuous inverse. The inverse function theorem then ensures that

$$
\Pi_{\mid \mathcal{N}}^{-1}: \mathcal{Q} \rightarrow \mathcal{N}
$$

is well-defined and analytic. We get the desired result by observing that

$$
N=\Pi_{\mid \mathcal{N}}^{-1} \circ \Pi \text {. }
$$




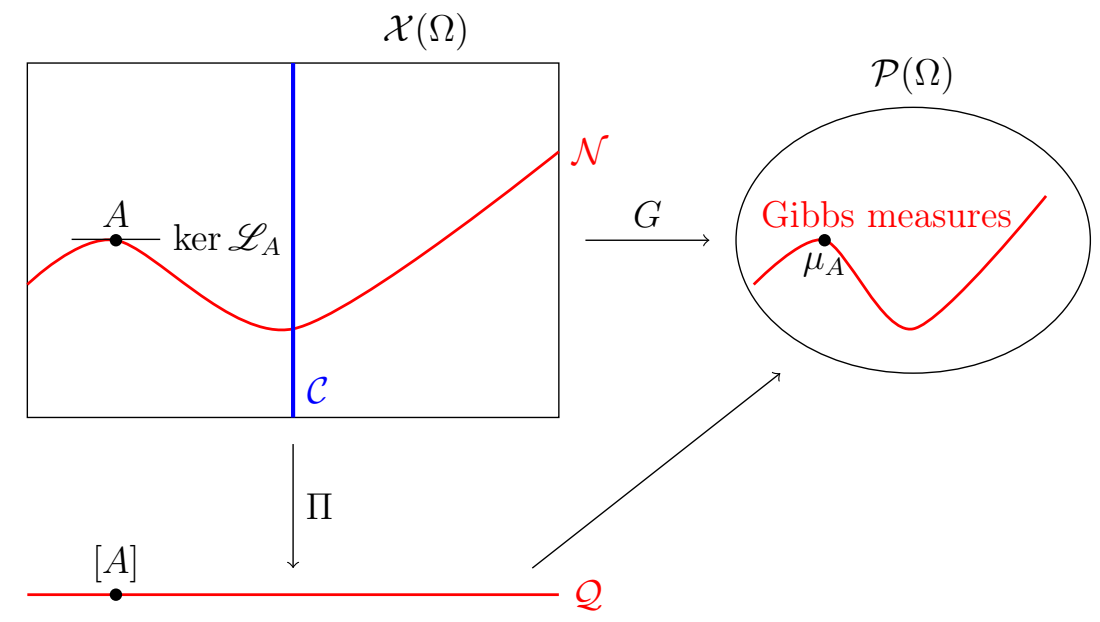

Figure 1: Potentials and Gibbs measures

Corollary 3.6. The maps $\Lambda: \mathcal{X}(\Omega) \rightarrow \mathbb{R}$ and $H: \mathcal{X}(\Omega) \rightarrow \mathcal{X}(\Omega)$ sending a potential to its leading eigendata, i.e. defined by

$$
\Lambda(A)=\lambda_{A} \quad \text { and } \quad H(A)=h_{A}
$$

(normalized by the condition $\log h_{A} \in \operatorname{ker} \mu_{A_{0}}$ for any fixed $A_{0}$ ) are analytic maps.

Moreover for all $A, \zeta \in \mathcal{X}(\Omega)$ :

$$
D(\log \Lambda)_{A}(\zeta)=\int \zeta \mathrm{d} \mu_{A}
$$

Note that it will turn out that in our framework $\log \Lambda$ equals the pressure functional, so that this result gives also the derivative of the later.

Proof. Fix $A_{0}$ be any potential, which can be assumed without loss of generality to be normalized. We then have

$$
\Lambda(A)=\exp \left(\int(A-N(A)) \mathrm{d} \mu_{A_{0}}\right) \quad \text { and } \quad H(A)=\exp \left(\mathscr{M}_{A_{0}}(A-N(A))\right.
$$

which are analytic as composed of analytic maps.

Differentiating $\log \Lambda(A)=\int(A-N(A)) \mathrm{d} \mu_{A_{0}}$ with respect to $A$ it comes

$$
D(\log \Lambda)_{A}(\zeta)=\int\left(\zeta-D N_{A}(\zeta)\right) \mathrm{d} \mu_{A_{0}} .
$$

This holds for any $A_{0}$ and any $A$, in particular taking $A_{0}=A$ and observing that $D N_{A}(\zeta) \in \operatorname{ker} \mu_{A}$ yields the desired formula.

Corollary 3.7. The map $G: A \mapsto \mu_{A} \in \mathcal{X}(\Omega)^{*}$ is analytic. In particular for each $\varphi \in \mathcal{X}(\Omega)$, the map $G_{\varphi}: A \mapsto \int \varphi \mathrm{d} \mu_{A}$ is analytic. 
Proof. Corollary 3.6 implies that $\mu_{A}=D(\log \Lambda)_{A}$ as a linear form defined on $\mathcal{X}(\Omega)$, so that the Corollary follows from the analyticity of $\log \Lambda$.

At this point we have proved Corollary B from the introduction.

Corollaries 3.6 and 3.7 where obtained under different assumptions and with different methods by Bomfim, Castro and Varandas [BCV12]; note that we notably do not assume the high-temperature regime (see their conditions $(\mathrm{P})$ and $\left(\mathrm{P}^{\prime}\right)$ ) and that once our framework is set, our proofs are very simple.

\section{Differentiating the Gibbs map in the affine structure}

There are at least two ways to endow the set of probability measures $\mathcal{P}(\Omega)$ with a kind of differential structure, i.e. to define what it means for a map such as the Gibbs map $G: A \mapsto \mu_{A}$ to be differentiable. In this section, we consider the affine structure, while in Section 6 we will consider the Wasserstein structure.

The affine structure is obtained simply by observing that $\mathcal{P}(\Omega)$ is a convex set in $\mathcal{X}(\Omega)^{*}$; "coordinates" are obtained by looking at integral of test functions, so that $G$ is often considered to be differentiable if

$$
\forall A, \zeta, \varphi \in \mathcal{X}(\Omega):\left.\quad \frac{\mathrm{d}}{\mathrm{d} t} \int \varphi \mathrm{d} \mu_{A+t \zeta}\right|_{t=0} \text { exists. }
$$

We will adopt here the definition of Fréchet differentiability for $G: \mathcal{X}(\Omega) \rightarrow \mathcal{X}(\Omega)^{*}$. It is stronger than the above one in three respects: we ask that for each $\varphi$ the directional derivatives at $A$ can be collected as a continuous linear map $\mathcal{X}(\Omega) \rightarrow \mathbb{R}$, that all these linear maps for various $\varphi$ can be collected as a continuous linear map $\mathcal{X}(\Omega) \rightarrow \mathcal{X}(\Omega)^{*}$, and that in the Taylor formula defining the derivative, the remainder is of the form $o(\|\varphi\|\|\zeta\|)$ (when $\zeta \rightarrow 0$ ). Note that at this point this strong definition is already ensured by the analyticity of $G$ and we only want to get an explicit formula.

Theorem 4.1. For all $A \in \mathcal{X}(\Omega)$ there is a neighborhood $U$ of 0 in $\mathcal{X}(\Omega)$ such that for all $\varphi \in \mathcal{X}(\Omega)$ and all $\zeta \in U$, we have

$$
\int \varphi \mathrm{d} \mu_{A+\zeta}-\int \varphi \mathrm{d} \mu_{A}=\int\left(I-\mathscr{L}_{N(A)}\right)^{-1}\left(\varphi_{A}\right) \cdot D N_{A}(\zeta) \mathrm{d} \mu_{A}+O\left(\|\varphi\|\|\zeta\|^{2}\right)
$$

where $\varphi_{A}:=\varphi-\int \varphi \mathrm{d} \mu_{A}$ is the projection of $\varphi$ on ker $\mu_{A}$ along the space of constants.

Implicitly, the constant in the $O$ depends only on $A$ (and of course $U, \Omega, T, \mathcal{X}(\Omega)$ ) but not on $\varphi$ and $\zeta$. This result will be deduced from the following special case where the expression is simpler.

Theorem 4.2. Assume that $A$ is normalized, $\varphi$ has mean 0 with respect to $\mu_{A}$, and $\zeta$ is tangent to $\mathcal{N}$ at $A$ and small enough. Then:

$$
\int \varphi \mathrm{d} \mu_{A+\zeta}=\int\left(I-\mathscr{L}_{A}\right)^{-1}(\varphi) \cdot \zeta \mathrm{d} \mu_{A}+O\left(\|\varphi\|\|\zeta\|^{2}\right)
$$


Writing $G_{\varphi}$ the composition of the evaluation at $\varphi$ and the Gibbs map, i.e. $G_{\varphi}(A)=$ $\int \varphi \mathrm{d} \mu_{A}$, the above formula can be recast as:

$$
D\left(G_{\varphi}\right)_{A}(\zeta)=\int\left(I-\mathscr{L}_{A}\right)^{-1}(\varphi) \cdot \zeta \mathrm{d} \mu_{A} \quad \text { when } A \in \mathcal{N}, \zeta \in \operatorname{ker} \mathscr{L}_{A}, \varphi \in \operatorname{ker} \mu_{A} .
$$

Observe that using the series expression of $\left(I-\mathscr{L}_{A}\right)^{-1}$, that $\mu_{A}$ is fixed by $\mathscr{L}_{A}^{*}$ and that the transfer operator is a left-inverse to the composition operator, this also rewrites as

$$
D\left(G_{\varphi}\right)_{A}(\zeta)=\sum_{i=0}^{+\infty} \int \varphi \cdot \zeta \circ T^{i} \mathrm{~d} \mu_{A}
$$

This version has the advantage that it applies to test functions $\varphi$ not necessarily in ker $\mu_{A}$, because $\zeta \in \operatorname{ker} \mathscr{L}_{A}$ implies $\zeta \in \operatorname{ker} \mu_{A}$ and adding a constant to $\varphi$ does therefore not change the value of the integrals.

We can rephrase Theorem 4.1 in a similar way, which will be used in the sequel to define a metric on $\mathcal{X}(\Omega)$.

Corollary 4.3. For all $A, \zeta, \varphi \in \mathcal{X}(\Omega)$, if $\zeta$ is small enough we have

$$
\begin{aligned}
& \int \varphi \mathrm{d} \mu_{A+\zeta}-\int \varphi \mathrm{d} \mu_{A}= \\
& \qquad \varphi_{A} \zeta \mathrm{d} \mu_{A}+\sum_{i=1}^{\infty} \int\left(\varphi_{A} \cdot \zeta \circ T^{i}+\varphi_{A} \circ T^{i} \cdot \zeta\right) \mathrm{d} \mu_{A}+O\left(\|\varphi\|\|\zeta\|^{2}\right)
\end{aligned}
$$

where the above sum converges and defines a continuous bilinear form.

\subsection{The case of a pair of normalized potentials}

To obtain Theorem 4.2, thanks to the regularity of the normalization map proved in the previous section, we are mostly reduced to estimate $\int \varphi \mathrm{d}\left(\mu_{B}-\mu_{A}\right)$ when $\varphi \in \mathcal{X}(\Omega)$ is fixed and $A, B$ are normalized potentials. Up to adding a constant to $\varphi$, which does not change the value of the above integral, we assume that $\varphi \in \operatorname{ker} \mu_{A}$.

We first write (using that $\mu_{A}$ and $\mu_{B}$ are respectively fixed by $\mathscr{L}_{A}^{*}$ and $\mathscr{L}_{B}^{*}$ )

$$
\begin{aligned}
\int \varphi \mathrm{d}\left(\mu_{B}-\mu_{A}\right) & =\int \mathscr{L}_{B}(\varphi) \mathrm{d} \mu_{B}-\int \mathscr{L}_{A}(\varphi) \mathrm{d} \mu_{A} \\
& =\int\left(\mathscr{L}_{B}(\varphi)-\mathscr{L}_{A}(\varphi)\right) \mathrm{d} \mu_{B}+\int \mathscr{L}_{A}(\varphi) \mathrm{d}\left(\mu_{B}-\mu_{A}\right)
\end{aligned}
$$

Then, we observe

$$
\begin{aligned}
\left(\mathscr{L}_{B}(\varphi)-\mathscr{L}_{A}(\varphi)\right)(x) & =\sum_{T(y)=x} e^{A(y)} \varphi(y)\left(e^{B(y)-A(y)}-1\right) \\
& =\mathscr{L}_{A}\left(\varphi\left(e^{B-A}-1\right)\right)(x)
\end{aligned}
$$


so that writing $R(x)=e^{x}-1-x \sim \frac{1}{2} x^{2}$, we get

$$
\mathscr{L}_{B}(\varphi)-\mathscr{L}_{A}(\varphi)=\mathscr{L}_{A}(\varphi \cdot(B-A))+\mathscr{L}_{A}(\varphi \cdot R(B-A))
$$

Thus:

$$
\begin{aligned}
\int \varphi \mathrm{d}\left(\mu_{B}-\mu_{A}\right)= & \int \mathscr{L}_{A}(\varphi \cdot(B-A)) \mathrm{d} \mu_{B}+\int \mathscr{L}_{A}(\varphi \cdot R(B-A)) \mathrm{d} \mu_{B} \\
& +\int \mathscr{L}_{A}(\varphi) \mathrm{d}\left(\mu_{B}-\mu_{A}\right) \\
= & \int \mathscr{L}_{A}(\varphi \cdot(B-A)) \mathrm{d} \mu_{A}+\int \mathscr{L}_{A}(\varphi \cdot(B-A)) \mathrm{d}\left(\mu_{B}-\mu_{A}\right) \\
& +\int \mathscr{L}_{A}(\varphi \cdot R(B-A)) \mathrm{d} \mu_{B}+\int \mathscr{L}_{A}(\varphi) \mathrm{d}\left(\mu_{B}-\mu_{A}\right) \\
\int \varphi \mathrm{d}\left(\mu_{B}-\mu_{A}\right)= & \int \cdot(B-A) \mathrm{d} \mu_{A}+\int \mathscr{L}_{A}(\varphi) \mathrm{d}\left(\mu_{B}-\mu_{A}\right) \\
& +\mathscr{I}(\varphi, B)
\end{aligned}
$$

where $\mathscr{I}(\varphi, B)=\int \mathscr{L}_{A}(\varphi \cdot(B-A)) \mathrm{d}\left(\mu_{B}-\mu_{A}\right)+\int \mathscr{L}_{A}(\varphi \cdot R(B-A)) \mathrm{d} \mu_{B}$, which is linear in $\varphi$ and which we now aim at bounding by a multiple of $\|\varphi\|\|B-A\|^{2}$.

A first tool is the regularity of $G$.

Lemma 4.4. The map $G: \mathcal{X}(\Omega) \rightarrow \mathcal{X}(\Omega)^{*}$ is locally Lipschitz: for all $A \in \mathcal{X}(\Omega)$ there exist a neighborhood $U \in \mathcal{X}(\Omega)$ of $A$ and a constant $C$ such that, for all $\varphi \in \mathcal{X}(\Omega)$ and all $B \in U$, it holds:

$$
\left|\int \varphi \mathrm{d}\left(\mu_{B}-\mu_{A}\right)\right| \leq C\|\varphi\|\|B-A\| .
$$

Proof. This follows from the analyticity of $G$ obtained in Corollary 3.7.

A second observation is that since $\mathcal{X}(\Omega)$ has a multiplicative norm, we get

$$
\begin{aligned}
\|R(B-A)\| & =\left\|\sum_{k \geq 2} \frac{1}{k !}(B-A)^{k}\right\| \\
& \leq \sum_{k \geq 2} \frac{1}{k !}\|B-A\|^{k} \\
& =R(\|B-A\|) \\
& \leq C^{\prime}\|B-A\|^{2}
\end{aligned}
$$

when $B$ is in any fixed neighborhood $U$ of $A$.

Now, since $\|\cdot\|$ is assumed to control the sup norm and $\mu_{B}$ is a probability measure, whenever $B \in U$ it comes

$$
\begin{aligned}
|\mathscr{I}(\varphi, B)| & \leq C\left|\mathscr{L}_{A}\right|\|\varphi\|\|B-A\|^{2}+C^{\prime \prime}\left|\mathscr{L}_{A}\right|\|\varphi\|\|R(B-A)\| \\
& \leq C^{\prime \prime \prime}\|\varphi\|\|B-A\|^{2}
\end{aligned}
$$


Now, applying (3) to its own second term repeatedly and recalling that $\mathscr{L}_{A}(\varphi)$ goes to zero thanks to the spectral gap assumption, we get

$$
\begin{aligned}
\int \varphi \mathrm{d}\left(\mu_{B}-\mu_{A}\right)= & \int \varphi \cdot(B-A) \mathrm{d} \mu_{A}+\int \mathscr{L}_{A}(\varphi) \mathrm{d}\left(\mu_{B}-\mu_{A}\right) \\
& +\mathscr{I}(\varphi, B) \\
= & \int\left(\varphi+\mathscr{L}_{A}(\varphi)\right) \cdot(B-A) \mathrm{d} \mu_{A}+\int \mathscr{L}_{A}^{2}(\varphi) \mathrm{d}\left(\mu_{B}-\mu_{A}\right) \\
& +\mathscr{I}\left(\varphi+\mathscr{L}_{A}(\varphi), B\right) \\
= & \int\left(\sum_{n \geq 0} \mathscr{L}_{A}^{n}(\varphi)\right) \cdot(B-A) \mathrm{d} \mu_{A}+\mathscr{I}\left(\sum_{n \geq 0} \mathscr{L}_{A}^{n}(\varphi), B\right) \\
= & \int\left(I-\mathscr{L}_{A}\right)^{-1}(\varphi) \cdot(B-A) \mathrm{d} \mu_{A}+O\left(\|\varphi\|\|B-A\|^{2}\right)
\end{aligned}
$$

which is almost Theorem 4.2, except for the assumption that $B$ is normalized.

\subsection{End of proofs}

Proof of Theorem 4.2. Since $N$ is an analytic projection to $\mathcal{N}$ (i.e. $N$ restricted to $\mathcal{N}$ is the identity), we have

$$
N(A+\zeta)=A+\zeta+O\left(\|\zeta\|^{2}\right)
$$

for all $A \in \mathcal{N}$ and all small enough $\zeta \in T_{A} \mathcal{N}=\operatorname{ker} \mathscr{L}_{A}$, with an implicit constant only depending on $A$.

Fix $A \in \mathcal{N}, \zeta \in \operatorname{ker} \mathscr{L}_{A}$ and $\varphi \in \operatorname{ker} \mu_{A}$, and set $B=N(A+\zeta)$. Using (4) with the normalized potentials $A$ and $B$, we get

$$
\begin{aligned}
\int \varphi \mathrm{d}\left(\mu_{A+\zeta}-\mu_{A}\right)= & \int \varphi \mathrm{d}\left(\mu_{A+\zeta}-\mu_{B}\right)+\int \varphi \mathrm{d}\left(\mu_{B}-\mu_{A}\right) \\
= & O(\|\varphi\|\|A+\zeta-N(A+\zeta)\|) \\
& \quad+\int\left(I-\mathscr{L}_{A}\right)^{-1}(\varphi) \cdot(B-A) \mathrm{d} \mu_{A}+O\left(\|\varphi\|\|B-A\|^{2}\right) \\
= & \int\left(I-\mathscr{L}_{A}\right)^{-1}(\varphi) \cdot \zeta \mathrm{d} \mu_{A}+O\left(\|\varphi\|\|\zeta\|^{2}\right),
\end{aligned}
$$

for $\zeta$ small enough, and with an implicit constant that depends only on $A$.

Proof of Theorem 4.1. Let $A, \zeta, \varphi \in \mathcal{X}(\Omega)$ be arbitrary. Then we consider:

- $N(A)$, which is the normalized potential such that $\mu_{N(A)}=\mu_{A}$,

- $D N_{A}(\zeta)$, which is the projection of $\zeta$ on $\operatorname{ker} \mathscr{L}_{N(A)}$ in the direction of $\mathcal{C}$,

- $\varphi_{A}=\varphi-\int \varphi \mathrm{d} \mu_{A} \in \operatorname{ker} \mu_{A}$ 
and we apply Theorem 4.2 to this new potential, tangent vector, and test function. We obtain exactly the desired expression once we notice that

$$
\begin{aligned}
\left|\mu_{A+\zeta}-\mu_{N(A)+D N_{A}(\zeta)}\right| & =\left|\mu_{N(A+\zeta)}-\mu_{N(A)+D N_{A}(\zeta)}\right| \\
& =O\left(\left\|N(A+\zeta)-N(A)-D N_{A}(\zeta)\right\|\right) \\
& =O\left(\|\zeta\|^{2}\right) .
\end{aligned}
$$

Proof of Corollary 4.3. We have to rewrite

$$
\int\left(I-\mathscr{L}_{N(A)}\right)^{-1} \varphi_{A} \cdot D N_{A}(\zeta) \mathrm{d} \mu_{A}
$$

We first observe that the final expression we aim for only involves $A$ through the measure $\mu_{A}$, so that we can as well replace $A$ by $N(A)$, i.e. assume that $A$ is normalized (this has for sole purpose to avoid writing a dozen times $\left.\mathscr{L}_{N(A)}\right)$.

We first write $\left(I-\mathscr{L}_{A}\right)^{-1} \varphi_{A}=\sum_{i>0} \mathscr{L}_{A}^{i} \varphi_{A}$, and recall that $D N_{A}(\zeta)$ is the projection of $\zeta$ to ker $\mathscr{L}_{A}$ along $\mathcal{C}$; this means that there is a function $g \in \mathcal{X}(\Omega)$ such that

$$
D N_{A}(\zeta)=\zeta_{A}+g-g \circ T
$$

(where $\left.\zeta_{A}=\zeta-\int \zeta \mathrm{d} \mu_{A} \in \operatorname{ker} \mu_{A}\right)$ and that $\mathscr{L}_{A}\left(D N_{A}(\zeta)\right)=0$. In particular, we have $\mathscr{M}_{A}\left(D N_{A}(\zeta)\right)=0 ;$ thus,

$$
g=\mathscr{M}_{A}\left(D N_{A}(\zeta)-\zeta_{A}\right)=-\mathscr{M}_{A}(\zeta)=\sum_{i \geq 1} \mathscr{L}_{A}^{i} \zeta_{A}
$$

This leads us to

$$
\begin{gathered}
\int\left(I-\mathscr{L}_{A}\right)^{-1} \varphi_{A} \cdot D N_{A}(\zeta) \mathrm{d} \mu_{A} \\
=\int \sum_{i \geq 0} \mathscr{L}_{A}^{i} \varphi_{A} \cdot \zeta_{A} \mathrm{~d} \mu_{A}+\int \sum_{i \geq 0} \mathscr{L}_{A}^{i} \varphi_{A} \cdot g \mathrm{~d} \mu_{A}-\int \sum_{i \geq 0} \mathscr{L}_{A}^{i} \varphi_{A} \cdot g \circ T \mathrm{~d} \mu_{A} \\
=\int \sum_{i \geq 0} \mathscr{L}_{A}^{i} \varphi_{A} \cdot \zeta_{A} \mathrm{~d} \mu_{A}+\int \sum_{i \geq 0} \mathscr{L}_{A}^{i} \varphi_{A} \cdot g \mathrm{~d} \mu_{A}-\int \sum_{i \geq 0} \mathscr{L}_{A}^{i+1} \varphi_{A} \cdot g \mathrm{~d} \mu_{A} \\
=\int \sum_{i \geq 0} \mathscr{L}_{A}^{i} \varphi_{A} \cdot \zeta_{A} \mathrm{~d} \mu_{A}+\int \varphi_{A} \cdot g \mathrm{~d} \mu_{A} \\
=\int \sum_{i \geq 0} \mathscr{L}_{A}^{i} \varphi_{A} \cdot \zeta_{A} \mathrm{~d} \mu_{A}+\int \varphi_{A} \cdot \sum_{i \geq 1} \mathscr{L}_{A}^{i} \zeta_{A} \mathrm{~d} \mu_{A},
\end{gathered}
$$

and, using the invariance of $\mu_{A}$ under $\mathscr{L}_{A}^{*}$ :

$$
\begin{aligned}
& \int\left(I-\mathscr{L}_{A}\right)^{-1} \varphi_{A} \cdot D N_{A}(\zeta) \mathrm{d} \mu_{A} \\
&=\int \varphi_{A} \zeta_{A} \mathrm{~d} \mu_{A}+\sum_{i \geq 1} \int\left(\varphi_{A} \cdot \zeta_{A} \circ T^{i}+\varphi_{A} \circ T^{i} \cdot \zeta_{A}\right) \mathrm{d} \mu_{A},
\end{aligned}
$$


where the sum converges (exponentially).

Finally, we observe that there is no use normalizing both $\varphi$ and $\zeta$, since for example $\int \varphi_{A} \zeta_{A} \mathrm{~d} \mu_{A}=\int \varphi_{A} \zeta \mathrm{d} \mu_{A}$. All $\zeta_{A}$ can therefore be replaced by $\zeta$, and we get the desired formula.

\section{A Riemannian metric on the space of normalized potentials}

The goal of this section is to define and to study a (weak) Riemannian metric on the space of Gibbs measures. More precisely, we construct a Riemannian metric on the manifold of normalized potentials, which corresponds equivalently to a Riemannian metric on the quotient space $\mathcal{Q}=\mathcal{X}(\Omega) / \mathcal{C}$, and relates in various ways to dynamical quantities. After a conformal rescaling by the metric entropy, this metric is very closely related to the metric defined by McMullen [McM08] (see also [BCS15] and references therein).

\subsection{Weak and strong inner products on Banach spaces}

Consider a positive symmetric bilinear form $\langle\cdot, \cdot\rangle$ on some Banach space $\mathcal{Y}$. There are two possible definitions of positive-definiteness. The first one is a copy and paste of the finite-dimensional definition, that is, we ask that

$$
\forall y \neq 0 \in \mathcal{Y}: \quad\langle y, y\rangle>0 .
$$

In this case, one says that $\langle\cdot, \cdot\rangle$ is weakly positive-definite. The second one is to ask that the Banach norm $\|\cdot\|$ of $\mathcal{Y}$ controls $\langle\cdot, \cdot\rangle$ from below, that is,

$$
\exists C>0, \forall y \in \mathcal{Y}: \quad\langle y, y\rangle \geq C\|y\|^{2}
$$

In this case, one says that $\langle\cdot, \cdot\rangle$ is strongly positive-definite; note that this condition implies weak positive-definiteness.

Most of the time, one is only interested in bilinear forms which are continuous with respect to the Banach topology of $\mathcal{Y}$. But if $\langle\cdot, \cdot\rangle$ is both continuous and strongly positive-definite, then its associated norm is equivalent to $\|\cdot\|$, and in particular $\mathcal{Y}$ must be isomorphic to a Hilbert space. Therefore, most Banach spaces have no continuous, strongly positive-definite inner product.

We shall say that $\langle\cdot, \cdot\rangle$ is an inner product if it is continuous and weakly positivedefinite, and use the term semi-definite inner product for a merely continuous, positive semi-definite symmetric bilinear form. By a Riemannian metric on a smooth Banach manifold, we mean a field of inner products on the tangent spaces, such that when translated in a chart, the inner product depends smoothly on the point, that is, it defines a smooth map from the domain of the chart to the Banach space of symmetric bilinear forms.

As a last remark, note that when $\langle\cdot, \cdot\rangle$ is an inner product inducing a complete norm, it endows $\mathcal{Y}$ with a second structure of Banach space (more precisely a Hilbert structure 
of course). Then the the identity map $\mathcal{Y} \rightarrow \mathcal{Y}$ is a continuous bijection between the two Banach structures at hand, and is therefore an isomorphism. This implies in particular that $\langle\cdot, \cdot\rangle$ is strongly positive-definite. In other words, inner products which are not strongly positive-definite induce a norm which is never complete. This means that there will be a relatively subtle interplay between the topology of $\mathcal{Y}$ and the measurements made from $\langle\cdot, \cdot\rangle$.

\subsection{The Variance metric}

Now, we introduce our proposed metric. Its main properties are summed up in the following result.

Theorem 5.1. There exists an analytic map from $\mathcal{X}(\Omega)$ to the space of its continuous symmetric bilinear forms, which maps any potential $A$ to a semi-definite inner product $\langle\cdot, \cdot\rangle_{A}$ such that:

1. $\langle\cdot, \cdot\rangle_{A}$ restricts to $T_{A} \mathcal{N}$ into an inner product for all $A \in \mathcal{N}$, thus inducing a Riemannian metric on $\mathcal{N}$,

2. this Riemannian metric coincides with the one obtained from $L^{2}\left(\mu_{A}\right)$ :

$$
\forall A \in \mathcal{N}, \forall \eta, \zeta \in T_{A} \mathcal{N}: \quad\langle\eta, \zeta\rangle_{A}=\int \eta \zeta \mathrm{d} \mu_{A},
$$

3. for all $A,\langle\cdot, \cdot\rangle_{A}$ induces a well defined inner product on $\mathcal{Q}$, thus inducing a Riemannian metric on this quotient space,

4. for all $A, \zeta, \varphi \in \mathcal{X}(\Omega)$, it holds:

$$
\left.\frac{\mathrm{d}}{\mathrm{d} t} \int \varphi \mathrm{d} \mu_{A+t \zeta}\right|_{t=0}=\langle\varphi, \zeta\rangle_{A}
$$

5. for all $A, \zeta \in \mathcal{X}(\Omega)$, it holds:

$$
\operatorname{Var}\left(\zeta_{A}, \mu_{A}\right):=\lim \frac{1}{n} \int\left(\sum_{i=0}^{n-1} \zeta_{A} \circ T^{i}\right)^{2} \mathrm{~d} \mu_{A}=\langle\zeta, \zeta\rangle_{A} .
$$

Of course, the metrics in $\mathcal{N}$ and $\mathcal{X}(\Omega) / \mathcal{C}$ correspond one to the other through the natural identification between these two spaces. There is really only one Riemannian metric, which can be viewed in two ways. Any of the last two items completely specify $\langle\cdot, \cdot\rangle_{A}$, and can be taken as a definition. Our point here is that these expressions define the same bilinear form, inducing an inner product on $T_{A} \mathcal{N}$.

The end of this section is devoted to the proof of Theorem 5.1. The first step leading to this result is to observe that the expression in Corollary 4.3 is symmetric: in the right-hand side, $\zeta$ and $\varphi$ play the same role (up to normalization, but the formula holds 
and was even proved with $\zeta$ also normalized to $\zeta_{A}$ ). This means that the function against which $\mu_{A}$ is tested and the direction in which $A$ is moved play precisely the same role in the evolution of the integral, a somewhat surprising connection (but which also follows from Corollary 3.6 and the Schwarz Lemma). It also indicates that the right-hand side in Corollary 4.3 defines a symmetric bilinear form: given $A \in \mathcal{X}(\Omega)$, we define for all $\eta, \zeta \in \mathcal{X}(\Omega)$ :

$$
\begin{aligned}
\langle\eta, \zeta\rangle_{A} & =\int \eta_{A} \cdot \zeta_{A} \mathrm{~d} \mu_{A}+\sum_{i \geq 1} \int\left(\eta_{A} \cdot \zeta_{A} \circ T^{i}+\eta_{A} \circ T^{i} \cdot \zeta_{A}\right) \mathrm{d} \mu_{A} \\
& =\int \eta_{A} \cdot \zeta_{A} \mathrm{~d} \mu_{A}+\sum_{i \geq 1} \int\left(\mathscr{L}_{N(A)}^{i}\left(\eta_{A}\right) \cdot \zeta_{A}+\eta_{A} \cdot \mathscr{L}_{N(A)}^{i}\left(\zeta_{A}\right)\right) \mathrm{d} \mu_{A}
\end{aligned}
$$

The second expression shows that $\langle\eta, \zeta\rangle_{A}$ is a well defined number, and that it defines a continuous symmetric bilinear form on $\mathcal{X}(\Omega)$. It also follows from Section 4 that $\langle\cdot, \cdot\rangle_{A}$ depends continuously on $A$; in fact the analyticity of $\langle\cdot, \cdot\rangle_{A}$ follows from Corollary 3.6 in the same way as Corollary 3.7: we have $\langle\zeta, \eta\rangle_{A}=D^{2}(\log \Lambda)_{A}(\zeta, \eta)$ which depends analytically on $A$.

From Corollary 4.3, we see that

$$
\langle\eta, \zeta\rangle_{A}=\int\left(I-\mathscr{L}_{N(A)}\right)^{-1}\left(\eta_{A}\right) \cdot D N_{A}(\zeta) \mathrm{d} \mu_{A},
$$

which seems asymmetric but will be useful.

As it is, $\langle\cdot, \cdot\rangle_{A}$ does not define an inner product, because it is not weakly positivedefinite.

Proposition 5.2. The symmetric form $\langle\cdot, \cdot,\rangle_{A}$ is positive semi-definite, and for all $A \in$ $\mathcal{N}$ and $\zeta \in T_{A} \mathcal{N}$ we have $\langle\zeta, \zeta\rangle_{A}=\int \zeta^{2} \mathrm{~d} \mu_{A}$. Moreover given $A, \zeta \in \mathcal{X}(\Omega)$, the following three statements are equivalent:

1. $\langle\zeta, \eta\rangle_{A}=0$, for all $\eta \in \mathcal{X}(\Omega)$,

2. $\langle\zeta, \zeta\rangle_{A}=0$,

3. $\zeta \in \mathcal{C}$.

Proof. First, observe that when $A \in \mathcal{N}$ and $\zeta \in T_{A} \mathcal{N}$ we have $\zeta_{A}=\zeta$ and $\mathscr{L}_{A}(\zeta)=0$, so that:

$$
\langle\zeta, \zeta\rangle_{A}=\int \zeta_{A} \cdot \zeta_{A} \mathrm{~d} \mu_{A}+\sum_{i \geq 1} \int\left(\mathscr{L}_{A}^{i}\left(\zeta_{A}\right) \cdot \zeta_{A}+\zeta_{A} \cdot \mathscr{L}_{A}^{i}\left(\zeta_{A}\right)\right) \mathrm{d} \mu_{A}=\int \zeta \cdot \zeta \mathrm{d} \mu_{A}
$$

It is clear that 1 implies 2 , and (6) shows that 3 implies 1 . Let us show that 2 implies 3. Since $\langle\cdot, \cdot\rangle_{A}$ does not change if we add a constant or a coboundary to $A$ (i.e. it only depends on $\mu_{A}$ ), we can assume that $A$ is normalized.

Suppose that $\zeta$ is isotropic, i.e. $\langle\zeta, \zeta\rangle_{A}=0$. By Proposition 3.3, we decompose $\zeta=\zeta^{\prime}+f$, where $\zeta^{\prime} \in \operatorname{ker} \mathscr{L}_{A}$ and $f \in \mathcal{C}$. Then, $\left\langle\zeta^{\prime}, \zeta^{\prime}\right\rangle_{A}=\langle\zeta, \zeta\rangle_{A}$, since $\mathcal{C}$ is in the kernel of $\langle\cdot, \cdot\rangle_{A}$. Thus, we get $0=\left\langle\zeta^{\prime}, \zeta^{\prime}\right\rangle_{A}=\int \zeta^{\prime 2} \mathrm{~d} \mu_{A}$. It follows that $\zeta^{\prime}=0$ and $\zeta=f \in \mathcal{C}$. 
The last line of this proof is where we use (H5).

Definition 5.3. Let $A \in \mathcal{X}(\Omega)$ be any potential and $[A] \in \mathcal{Q}=\mathcal{X}(\Omega) / \mathcal{C}$ be its class modulo $\mathcal{C}$. For all $[\eta],[\zeta] \in \mathcal{Q}$, we define

$$
\langle[\eta],[\zeta]\rangle_{[A]}=\langle\eta, \zeta\rangle_{A},
$$

which is well-defined by Proposition 5.2, i.e. does not depend on the chosen representatives in each class. If $A \in \mathcal{N}$, we still write $\langle\cdot, \cdot\rangle_{A}$ for the restriction of this inner product to $T_{A} \mathcal{N}$. Proposition 5.2 shows that both these products are weakly positive-definite, and thus induce a norm on the Banach space they are defined on $\left(\mathcal{Q}\right.$ and $T_{A} \mathcal{N}=\operatorname{ker} \mathscr{L}_{A}$, respectively). We denote both norms by $\|\cdot\|_{A}$, i.e.

$$
\|\zeta\|_{A}=\sqrt{\langle\zeta, \zeta\rangle_{A}}
$$

and we use this notation for general $\zeta \in \mathcal{X}(\Omega)$.

Let us now prove the last statement of Theorem 5.1. As usual, we can define the variance of a function in $\operatorname{ker} \mu_{A}$ by

$$
\operatorname{Var}\left(\zeta_{A}, \mu_{A}\right):=\lim \frac{1}{n} \int\left(\sum_{i=0}^{n-1} \zeta_{A} \circ T^{i}\right)^{2} \mathrm{~d} \mu_{A} .
$$

By direct computation, we obtain

$$
\begin{aligned}
\int\left(\sum_{i=0}^{n} \zeta_{A} \circ T^{i}\right)^{2} \mathrm{~d} \mu_{A} & =\sum_{i, j=0}^{n-1} \int \zeta_{A} \circ T^{i} \cdot \zeta_{A} \circ T^{j} \mathrm{~d} \mu_{A} \\
& =n \int \zeta_{A}^{2} \mathrm{~d} \mu_{A}+2 \sum_{0 \leq i<j \leq n-1} \int \zeta_{A} \cdot \zeta_{A} \circ T^{j-i} \mathrm{~d} \mu_{A} \\
& =n \int \zeta_{A}^{2} \mathrm{~d} \mu_{A}+2 \sum_{k=1}^{n-1}(n-k) \int \zeta_{A} \cdot \zeta_{A} \circ T^{k} \mathrm{~d} \mu_{A} .
\end{aligned}
$$

Assume without loss of generality that $A$ is normalized. Then,

$$
\begin{gathered}
\frac{1}{n} \int\left(\sum_{i=0}^{n-1} \zeta_{A} \circ T^{i}\right)^{2} \mathrm{~d} \mu_{A}=\int \zeta_{A}^{2} \mathrm{~d} \mu_{A}+2 \sum_{k=1}^{n-1} \int \zeta_{A} \cdot \zeta_{A} \circ T^{k} \mathrm{~d} \mu_{A} \\
-\frac{2}{n} \sum_{k=1}^{n-1} \int k \mathscr{L}_{A}^{k}\left(\zeta_{A}\right) \cdot \zeta_{A} \mathrm{~d} \mu_{A},
\end{gathered}
$$

where the last term is bounded in norm by $O\left(\frac{\left\|\zeta_{A}\right\|^{2}}{n} \sum_{k=1}^{\infty} k \delta^{k}\right)$ with $\delta$ the spectral gap of $\mathscr{L}_{A}$.

It follows that for all $A, \zeta$ we have

$$
\operatorname{Var}\left(\zeta_{A}, \mu_{A}\right)=\|\zeta\|_{A}^{2}
$$


which, as usual in common examples and as follows from Proposition 5.2, vanishes exactly when $\zeta \in \mathcal{C}$.

This concludes the proof of Theorem 5.1, and of Theorem D once one observes that

$$
D^{2}(\log \Lambda)_{A}=D G_{A}=\langle\cdot, \cdot\rangle_{A}
$$

Remark 5.4. We have thus recovered in our setting the convexity of $\log \Lambda$, which will be given - as is customary - an interpretation in term of "pressure" below.

A notable consequence of this is that the submanifold $\mathcal{N}$ does not contain any straight interval: it is contained in the zero level set of $\log \Lambda$, so that any straight interval in $\mathcal{N}$ passing through $A$ would have its direction in $T_{A} \mathcal{N}$ and in the kernel of $\langle\cdot, \cdot\rangle_{A}$, whose intersection is trivial.

\section{Regularity of the Gibbs map: the Wasserstein structure}

The development of optimal transportation and more precisely of the 2-Wasserstein distance has let an alternative differential structure for the set $\mathcal{P}(\Omega)$ emerge, notably driven by the work of Otto [Ott01], Benamou and Brenier [BB00] and Ambrosio, Gigli and Savaré [AGS05]. We shall rely on the formulation given by [Gig11], which allows to define the differentiability of a map at a point (as opposed to more global notions, such as speed vectors defined almost everywhere). One could in principle consider the case when $\Omega$ is a Riemannian manifold, but for simplicity we shall restrict to $\Omega=\mathbb{S}=\mathbb{R} / \mathbb{Z}$ throughout this section.

\subsection{Elements of optimal transportation}

We will not give much details on optimal transportation, but many references are available (e.g. [Vil09] for a comprehensive source). Let us say that the 2-Wasserstein distance $W_{2}$ is a metric compatible with the weak topology, defined on $\mathcal{P}(\Omega)$ as the least cost needed to move one measure to another, when the cost to move a unit of mass is proportional to the squared distance between the starting point and the stopping point.

For each $\mu \in \mathcal{P}\left(\mathbb{S}^{1}\right)$, Gigli introduces a tangent space $T_{\mu} \mathcal{P}\left(\mathbb{S}^{1}\right)$ which may be only a metric cone, but turns out to be a Hilbert space in a number of cases. There are several possible definitions of such a tangent space (or cone), e.g. in term of geodesics, in term of measures on the tangent bundle, or in term of vector fields on the manifold; the work of Gigli ties all these points of view together when $\mu$ belongs to a certain class of "nice" measures. In the present one-dimensional case, the relevant class to be considered is the set of atomless measures. Assuming $\mu \in \mathcal{P}\left(\mathbb{S}^{1}\right)$ has no atom, one can consider as tangent space to $\mathcal{P}\left(\mathbb{S}^{1}\right)$ at $\mu$ the space

$$
T_{\mu} \mathcal{P}\left(\mathbb{S}^{1}\right):=L_{\nabla}^{2}(\mu):={\overline{\left\{\nabla f \mid f \in C^{\infty}\left(\mathbb{S}^{1}, \mathbb{R}\right)\right\}}}^{L^{2}(\mu)}
$$


of vector fields on $\mathbb{S}^{1}$ which are square-integrable with respect to $\mu$ and which are limits of gradients of smooth function in $L^{2}(\mu)$ (note that the quotient structure of $\mathbb{S}^{1}=\mathbb{R} / \mathbb{Z}$ makes it possible to identify all tangent spaces of $\mathbb{S}^{1}$ with $\mathbb{R}$, so that we can see vector fields as functions, and $\nabla f$ is simply $\left.f^{\prime}\right)$. There is an obvious exponential map: given $\mu$ and $v \in T_{\mu} \mathcal{P}\left(\mathbb{S}^{1}\right)$ one sets $\exp _{\mu}(v)=(\operatorname{Id}+v)_{\#} \mu$, i.e. the mass at any point $x \in \mathbb{S}^{1}$ is moved to $x+v(x) \bmod 1$. Then for each $v \in T_{\mu} \mathcal{P}\left(\mathbb{S}^{1}\right)$, one gets an exponential curve $\left(\exp _{\mu}(t v)\right)_{t \in[0, \varepsilon)}$ which has the property that

$$
W_{2}\left(\mu, \exp _{\mu}(t v)\right)=t\|v\|_{\mu}+o(t)
$$

where $\|v\|_{\mu}$ is the $L^{2}(\mu)$-norm of $v$ (here the fact that $v$ can be approximated by gradients is crucial).

We will say that a curve $t \mapsto \mu_{t}$ from an interval to $\mathcal{P}\left(\mathbb{S}^{1}\right)$ is Wasserstein-differentiable at $t_{0}$ with tangent vector $v \in T_{\mu_{0}} \mathcal{P}\left(\mathbb{S}^{1}\right)$ whenever it holds

$$
W_{2}\left(\mu_{t_{0}+h}, \exp _{\mu_{t_{0}}}(h v)\right)=o(h) .
$$

Similarly, a map $H: \mathcal{Y} \rightarrow \mathcal{P}\left(\mathbb{S}^{1}\right)$ from a Banach space to the set of probability measures on $\mathbb{S}^{1}$ is Wasserstein-differentiable at a point $A \in \mathcal{Y}$ in a direction $\zeta \in \mathcal{Y}$ whenever there exist $v \in T_{\mu} \mathcal{P}\left(\mathbb{S}^{1}\right)$ such that

$$
W_{2}\left(H(A+t \zeta), \exp _{H(A)}(t v)\right)=o(t)
$$

i.e. the tangent vector $v$ describes the first-order variations of $H$ in the Wassertein distance. Of course, one can define more stringent versions of this definition (Fréchetlike rather than Gâteaux-like), but since our result is negative we get the strongest statement by sticking to the weakest definition.

When $\Omega$ is a manifold, in each of its variations (Gâteaux or Fréchet), Wasserstein differentiablity is stronger than the corresponding variation of affine differentiability because of the continuity equation below; roughly, affine differentiability is about recording the "vertical" variations of the measure, i.e. the variation of weight it gives to any given set, while Wasserstein differentiability is about recording the "horizontal" variations of the measure, i.e. how one should move the mass in the most economical way in order to obtain the given change of measure. The physical principle of mass preservation leads to the continuity equation, which in the present case $\Omega=\mathbb{S}^{1}$ has the following form:

Lemma 6.1. Assume that $\left(\mu_{t}\right)_{t}$ is a curve of probability measures on $\mathbb{S}^{1}$ which is differentiable at 0 with tangent vector $v \in T_{\mu_{0}} \mathcal{P}\left(\mathbb{S}^{1}\right)$, then for all smooth function $\varphi$ we have

$$
\left.\frac{\mathrm{d}}{\mathrm{d} t} \int \varphi \mathrm{d} \mu_{t}\right|_{t=0}=\int \varphi^{\prime} v \mathrm{~d} \mu_{0} .
$$

The most common version of the continuity equation is stated for curves of measures, with the above equality integrated over time. The proof of the present version is very simple and can be found in [Klo15a]. 
A curve $\left(x_{t}\right)_{t \in I}$ in a metric space is said to be absolutely continuous whenever there is a positive function $g \in L^{1}(I)$ such that for all $t_{0}, t_{1} \in I$ :

$$
d\left(x_{t_{0}}, x_{t_{1}}\right) \leq \int_{t_{0}}^{t_{1}} g(s) \mathrm{d} s
$$

(note when considering a curve $\left(\mu_{t}\right)$ in $\mathcal{P}(\Omega)$, that this notion as nothing to do with each measure $\mu_{t}$ being absolutely continuous or not!) In other words, an absolutely continuous curve is a curve whose speed exists almost everywhere and is integrable. A particular case is given by Lipschitz curves, whose speed is in $L^{\infty}$; absolute continuity is therefore a very mild regularity condition. A Rademacher theorem holds in this setting: an absolutely continuous curve in $\mathcal{P}\left(\mathbb{S}^{1}\right)$ endowed with the 2-Wasserstein distance is differentiable at almost every time and satisfies the mean value theorem (see [AGS05]).

\subsection{Roughness of the Gibbs map in the Wasserstein space}

We are now in a position to state and prove the main result of this section, which shows that the Gibbs map is very far from being Wassertein-smooth.

Theorem 6.2. Assume $T$ is $x \mapsto d x \bmod 1$ acting on $\mathbb{S}^{1}$ and $\mathcal{X}\left(\mathbb{S}^{1}\right)$ is the space of $\alpha$-Hölder functions for some $\alpha \in(0,1]$. If $\left(A_{t}\right)_{t}$ is any smooth curve in $\mathcal{X}\left(\mathbb{S}^{1}\right)$, then its image curve $\left(\mu_{A_{t}}\right)_{t}$ under the Gibbs map is not (even locally) absolutely continuous in $\left(\mathcal{P}\left(\mathbb{S}^{1}\right), W_{2}\right)$ unless it is constant (i.e. unless $A_{t} \in A_{0}+\mathcal{C}$ for all $\left.t\right)$.

Recalling the interpretation of the Wasserstein metric $W_{2}$ above, we see that changing smoothly the potential changes smoothly the levels of the Gibbs measure (Theorem 4.1 ), but in a way that corresponds to brutal reallocations of the mass distribution (Theorem 6.2). This result should be compared to Corollary 1.3 in [KLS14], where a Lipschitz-regularity result is proved for the Gibbs map when $\mathcal{P}(\Omega)$ is endowed with the 1-Wasserstein distance (which however does not yield a differentiable structure).

The proof mostly relies on the following point-wise non-differentiability result.

Proposition 6.3. Under the same assumption as in Theorem 6.2, consider the Gibbs map $G: \operatorname{Hol}_{\alpha}\left(\mathbb{S}^{1}\right) \rightarrow \mathcal{P}\left(\mathbb{S}^{1}\right)$ sending each $A$ to $\mu_{A}$.

If $G$ is Wasserstein-differentiable at any potential $A$ in any direction $\zeta$, then either $\mu_{A}$ is the Lebesgue measure (i.e. $A \in \mathcal{C}$ ) or the derivative vanish (i.e. $\left.W_{2}\left(\mu_{A+t \zeta}, \mu_{A}\right)=o(t)\right)$.

Proof. Assume that $G$ is Wasserstein-differentiable at $A$ in the direction $\zeta$.

If $\varphi$ is any smooth function, on the one hand the continuity equation gives

$$
\left.\frac{\mathrm{d}}{\mathrm{d} t} \int \varphi \mathrm{d} \mu_{A+t \zeta}\right|_{t=0}=\int \varphi^{\prime} v \mathrm{~d} \mu_{A}
$$

where $v \in L^{2}\left(\mu_{A}\right)$ is some vector field (which can be approximated by gradients in $\left.L^{2}\left(\mu_{A}\right)\right)$; on the other hand section 4 gives

$$
\left.\frac{\mathrm{d}}{\mathrm{d} t} \int \varphi \mathrm{d} \mu_{A+t \zeta}\right|_{t=0}=\int\left(I-\mathscr{L}_{A}\right)^{-1}\left(\varphi_{A}\right) \cdot D N_{A}(\zeta) \mathrm{d} \mu_{A} .
$$


We get two very different-looking linear forms in $\varphi$ which both describe the variations of its integral. The proof will thus be complete as soon as we prove that unless $\mu_{A}$ is the Lebesgue measure, these two forms can agree only by vanishing.

For this, we use the following approximation lemma.

Lemma 6.4. Let $\mu$ be a measure on $\mathbb{S}^{1}$ which is singular with respect to the Lebesgue measure and without atoms; then for all $f \in L^{2}(\mu)$, and all $\beta<1$ there is a sequence of smooth functions $\varphi_{n}: \mathbb{S}^{1} \rightarrow \mathbb{R}$ such that $\varphi_{n}^{\prime} \rightarrow f$ in $L^{2}(\mu)$ and $\varphi_{n} \rightarrow 0$ in $\operatorname{Hol}_{\beta}\left(\mathbb{S}^{1}\right)$.

Proof. We first claim that when $I \subset[0,1]$ is an interval of length $\ell, w: I \rightarrow \mathbb{R}$ is measurable and $\mu$-essentially bounded by some number $M$, and $\varepsilon>0$, there is a smooth function $\varphi: I \rightarrow \mathbb{R}$ such that $\varphi$ and all its derivatives vanish at the endpoints of $I$, $\|\varphi\|_{\infty} \leq \varepsilon,\left\|\varphi^{\prime}\right\|_{\infty} \leq M$, and $\int_{I}\left(\varphi^{\prime}-w\right)^{2} \mathrm{~d} \mu \leq \varepsilon^{2} \ell^{2}$.

Let $\eta>0$ be arbitrary, to be chosen later on. Since $\mu$ is concentrated on a $\lambda$-negligible set, there is a finite set of intervals $I_{1}, \ldots, I_{k} \subset I$ with disjoint interiors whose total length is less than $\eta$ and whose complement $J=I \backslash\left(I_{1} \cup \cdots \cup I_{k}\right)$ is given by $\mu$ a mass less than $\eta$. Let $w_{1}$ be the function which:

- is constant on each $I_{i}$, with value the $\mu$-average of $w$ on $I_{i}$,

- is constant on $J$, with value such that $\int_{I} w_{1} \mathrm{~d} \lambda=0$.

By taking $\eta$ small enough and by dividing the intervals $I_{i}$ into smaller intervals, we can ensure that $\int\left(w-w_{1}\right)^{2} \mathrm{~d} \mu$ is arbitrarily small.

Let $w_{2}$ be a smooth approximation of $w_{1}$ such that $\int\left(w-w_{2}\right)^{2} \mathrm{~d} \mu$ stays small, $w_{2}$ is bounded by $M, \int_{I} w_{2}=0$, and $w_{2}$ is zero on some neighborhoods of the endpoint of $I$ (this last condition is easy to fulfill since $\mu$ has no atom).

Define a smooth, $M$-Lipschitz function $\varphi$ by

$$
\varphi(x):=\int_{a}^{x} w_{2}(t) \mathrm{d} t
$$

where $a=\min I$ is the starting point of $I$. Then $\varphi^{\prime}=w_{2}$ is close to $w$ in $L^{2}(\mu, I)$ norm and bounded above by $M$ (though $\varphi^{\prime \prime}$ is extremely large), and $\varphi$ and its derivatives vanish at both endpoints of $I$. The uniform norm of $\varphi$ is then bounded by $M \eta$, and can thus be made arbitrarily small, proving the claim.

Now, given $v$ and an integer $n$, choose a $\mu$-essentially bounded function $\bar{v}$ which is $1 / n$-close to $v$ in $L^{2}(\mu)$, call $M$ its essential bound, then choose $\ell$ small enough to ensure that $\ell^{1-\beta} M<1 / n$. Divide $\mathbb{S}^{1}$ into intervals of length $\ell$ and apply the claim to each of them. The boundary conditions enable us to glue the smooth functions defined on each interval into a smooth function $\varphi_{n}$ defined on $\mathbb{S}^{1}$, such that $\varphi_{n}^{\prime}$ is $M$-bounded and $1 / n$-close to $\bar{v}$ in $L^{2}(\mu)$ and $\left\|\varphi_{n}\right\|_{\infty}<1 / n$. For any $x, y \in \mathbb{S}^{1}$, when $|x-y| \leq \ell$ we get

$$
\frac{\left|\varphi_{n}(x)-\varphi_{n}(y)\right|}{|x-y|^{\beta}} \leq\left\|\varphi_{n}^{\prime}\right\||x-y|^{1-\beta} \leq M \ell^{1-\beta} \leq \frac{1}{n}
$$


and when $|x-y| \geq \ell$ we get

$$
\frac{\left|\varphi_{n}(x)-\varphi_{n}(y)\right|}{|x-y|^{\beta}} \leq \frac{\left|\varphi_{n}(x)-0\right|+\left|0-\varphi_{n}(y)\right|}{|x-y|^{\beta}} \leq \frac{2 M \ell}{|x-y|^{\beta}} \leq 2 M \ell^{1-\beta} \leq 2 / n .
$$

This proves the Lemma.

Now we simply apply the Lemma to $f=v$, and $\beta=\alpha$ if $\alpha<1$, or any lower $\beta$ otherwise (using that the thermodynamical formalism holds for the current $T$ with any $\beta)$. This gives us smooth functions $\varphi_{n}$ such that

$$
\int v^{2} \mathrm{~d} \mu_{A}=\left.\lim _{n} \frac{\mathrm{d}}{\mathrm{d} t} \int \varphi_{n} \mathrm{~d} \mu_{A+t \zeta}\right|_{t=0}=\int\left(I-\mathscr{L}_{A}\right)^{-1}(0) \cdot D N_{A}(\zeta) \mathrm{d} \mu_{A}=0
$$

(every operator being interpreted in the $\beta$-Hölder space if necessary) so that $v$ vanishes $\mu_{A}$-almost everywhere, and the Wasserstein derivative of $\mu_{A+t \zeta}$ vanishes.

Proof of Theorem 6.2. If $\left(\mu_{A_{t}}\right)_{t}$ is absolutely continuous, it is differentiable almost-everywhere and from Proposition 6.3 we deduce that at each $t$ such that $\mu_{A_{t}}$ is differentiable and not Lebesgue, its derivative vanishes. The mean value inequality then ensures that $\left(\mu_{A_{t}}\right)$ must then be constant.

We end this section with some open questions. First, Proposition 6.3 leaves open the following.

Question 6.5. In the case of $T: x \mapsto d x \bmod 1$, is the Gibbs map differentiable at $A$ when $A \in \mathcal{C}$ (i.e. when $\mu_{A}$ is the Lebesgue measure)?

Second, note that the analogue of Theorem 6.2 for the shift is true independently of the map $G$, since the 2-Wasserstein space of an ultrametric space such as $\mathcal{A}^{\mathbb{N}}$ contains no absolutely continuous curve at all (see [Klo15b]). But the 2-Wasserstein space of a manifold contains plenty of absolutely continuous curves (it is even a geodesic space), so when $\Omega$ has a smooth structure, the irregularity of $G$ with respect to the Wasserstein metric can be somewhat surprising. One then wonders how much it has to do with $G$, and how much it has to do with its image:

Question 6.6. Assume $\Omega$ is a manifold and $T$ is smooth. Are there any non-constant, absolutely continuous curves $\left(\mu_{t}\right)_{t}$ in $\left(\mathcal{P}(\Omega), W_{2}\right)$ such that $\mu_{t}$ is $T$-invariant for all $t$ ? What about the subset of Gibbs measures with $\alpha$-Hölder potential?

In other words, we ask whether the set of $T$-invariant measures is a nice, somewhat smooth subset of the set of all probability measures, or if from the Wasserstein point of view it is a very irregular subset of $\mathcal{P}\left(\mathbb{S}^{1}\right)$ (one can think of the Von Koch curve in $\mathbb{R}^{2}$ as an example of a connected, very irregular subset of a smooth space).

\section{Application to equilibrium states}

In this section we use the differential calculus developed above to study several classical optimization problems. 


\subsection{Entropy and pressure}

Given our broad framework, we shall use the following Legendre transform definition for entropy: for any $T$-invariant measure $\nu$, we set

$$
\mathrm{h}_{\mathcal{X}}(\nu):=\inf _{A \in \mathcal{X}}\left(\log \lambda_{A}-\int A \mathrm{~d} \nu\right) .
$$

Note that this quantity a priori depend on the chosen class of function $\mathcal{X}(\Omega)$; but in many cases it is in fact equal to the metric entropy of $\mu$, see remarks 7.5 and 7.8. The assumption (H6) ensures that $\mathcal{X}(\Omega)$ is quite large, preventing $\mathrm{h}_{\mathcal{X}}$ to be too degenerate.

Remark 7.1. The number $\log \lambda_{A}-\int A \mathrm{~d} \nu$ only depend on the class $[A]$ of $A$ modulo $\mathcal{C}$ (adding a constant to $A$ changes $\log \lambda_{A}$ and $\int A \mathrm{~d} \nu$ by the same additive constant, and adding a coboundary leaves both terms unchanged). In particular, one can rewrite

$$
\mathrm{h}_{\mathcal{X}}(\nu)=\inf _{A \in \mathcal{N}} \int(-A) \mathrm{d} \nu
$$

and observe that $A(y)=\log e^{A(y)}$ where

$$
\mathbb{P}(x \rightarrow y):= \begin{cases}e^{A(y)} & \text { when } T(y)=x \\ 0 & \text { otherwise }\end{cases}
$$

defines transition probabilities for a Markov chain on $\Omega$ supported on backward orbits of $T$. In other words, $H_{\nu}$ is the infimum of $\int(-\log \mathbb{P}(T(y) \rightarrow y)) \mathrm{d} \nu(y)$ over Markov chains supported on backward orbits of $T$, such that transition probabilities depends on the endpoint, with a regularity specified by $\mathcal{X}(\Omega)$.

Together with such a definition of entropy naturally comes a dual quantity, the pressure: for any potential $B \in \mathcal{X}(\Omega)$ we set

$$
\operatorname{Pr}(B):=\sup _{\mu \in \mathcal{P}_{T}(\Omega)}\left(\mathrm{h}_{\mathcal{X}}(\mu)+\int B \mathrm{~d} \mu\right)
$$

In many cases (e.g. shift in the Bernoulli space), this turns out to coincide with the classical topological pressure (see again remarks 7.5 and 7.8). Here we will concentrate on the study of the above Legendrian formulations for these quantities, as they fit our framework most naturally.

One of our main concern is to understand when and where the above infimum and supremum are attained; we thus consider the families of functionals defined for $\mu, \nu \in$ $\mathcal{P}_{T}(\Omega)$ and $A, B \in \mathcal{X}(\Omega)$ by

$$
\begin{aligned}
H_{\nu}(A) & =\log \lambda_{A}-\int A \mathrm{~d} \nu \\
P_{B}(\mu) & =\mathrm{h}_{\mathcal{X}}(\mu)+\int B \mathrm{~d} \mu
\end{aligned}
$$


The functional $P_{B}$ is defined for all $T$-invariant measures but we shall also study its restriction to Gibbs measures, considered as acting on potentials:

$$
P_{B}(A)=\mathrm{h}_{\mathcal{X}}\left(\mu_{A}\right)+\int B \mathrm{~d} \mu_{A}
$$

We will abusively use the same name $P_{B}$ for the map defined on invariant measure, the map defined on potentials, its restriction to normalized potential and the map it induces on the quotient $\mathcal{Q}=\mathcal{X}(\Omega) / \mathcal{C}$. The way we write the $\operatorname{argument}\left(P_{B}\left(\mu_{A}\right), P_{B}(A)\right.$ or $\left.P_{B}([A])\right)$ will usually make the difference clear.

Since $H_{\nu}$ is $\mathcal{C}$-invariant,it induces a functional on the quotient $\mathcal{Q}$, which we still denote by $H_{\nu}$.

\subsection{Classical equilibrium states and Legendre duality}

\subsubsection{The entropy functionals}

We start with the study of the functionals $H_{\nu}$.

Proposition 7.2. For all $\nu \in \mathcal{P}_{T}(\Omega)$, the functional $H_{\nu}$ on $\mathcal{X}(\Omega)$ is analytic with

$$
D\left(H_{\nu}\right)_{A}(\zeta)=\int \zeta \mathrm{d} \mu_{A}-\int \zeta \mathrm{d} \nu
$$

Moreover the map $[A] \mapsto H_{\nu}([A])$ induced on $\mathcal{Q}$ is strictly convex.

Proof. Let us recall that $\Lambda: \mathcal{X}(\Omega) \rightarrow(0,+\infty)$ is the analytic functional defined by $\Lambda(A)=\lambda_{A}$, and that for all $A, \zeta \in \mathcal{X}(\Omega)$ we have $D(\log \Lambda)_{A}(\zeta)=\int \zeta \mathrm{d} \mu_{A}$ (Corollary 3.6). Since the second term in $H_{\nu}(A)=\log \lambda_{A}-\int A \mathrm{~d} \nu$ is linear and thus analytic and equal to its derivative at any point, $H_{\nu}$ is analytic with $D\left(H_{\nu}\right)_{A}(\zeta)=\int \zeta \mathrm{d} \mu_{A}-\int \zeta \mathrm{d} \nu$. The second term is constant in $A$, and by the work of Sections 4 and 5 the second derivative is given by

$$
D^{2}\left(H_{\nu}\right)(\zeta, \eta)=D G_{A}(\eta)(\zeta)=\langle\eta, \zeta\rangle_{A}
$$

In other words, considering the functional $H_{\nu}$ induced on $\mathcal{Q}$ we have $D^{2}\left(H_{\nu}\right)=\langle\cdot, \cdot\rangle_{[A]}$ which is positive-definite, proving the strict convexity on $\mathcal{Q}$.

Note that we do not have uniform convexity (even locally) since the inner product is only weakly positive-definite (there are directions $[\zeta]$ with fixed size $\|[\zeta]\|$ such that the "convexity" $\|[\zeta]\|_{[A]}$ is arbitrarily small). Of course, $H_{\nu}$ is only weakly convex on $\mathcal{X}(\Omega)$ since it is constant along each fiber $A+\mathcal{C}$.

Proposition 7.2 now implies the following result.

Corollary 7.3. When $\nu=\mu_{B}$ for some $B \in \mathcal{X}(\Omega)$, then $H_{\mu_{B}}([A])$ is uniquely minimized at $[A]=[B]$ and thus

$$
\mathrm{h}_{\mathcal{X}}\left(\mu_{B}\right)=\log \lambda_{B}-\int B \mathrm{~d} \mu_{B}=-\int N(B) \mathrm{d} \mu_{B} .
$$

When $\nu$ is not in the image of the Gibbs map, $H_{\nu}$ does not reach its infimum. 
Note that a normalized $B$ is non-positive and non-zero and has $\lambda_{B}=1$, so that $\mathrm{h}_{\mathcal{X}}\left(\mu_{B}\right)>0$ (use (H5) to get the strict inequality).

Proof. Hypothesis $(\mathrm{H} 6)$ implies that $\mathcal{X}(\Omega)$ "separates measures" i.e.

$$
\left(\forall \zeta \in \mathcal{X}(\Omega): \int \zeta \mathrm{d} \mu=\int \zeta \mathrm{d} \nu\right) \Longrightarrow \mu=\nu
$$

Using $D\left(H_{\nu}\right)_{A}(\zeta)=\int \zeta \mathrm{d}\left(\mu_{A}-\nu\right)$ we see that when $\nu$ is not in the image of the Gibbs map $H_{\nu}$ has no critical point, hence no minimum; and when $\nu=\mu_{B}$ the critical points of $H_{\mu_{B}}$ are exactly the potentials $A$ such that $\mu_{A}=\mu_{B}$. Going down to the quotient we get only one critical point $[B]$ and the strict convexity implies that this critical point is the unique minimizer.

Remark 7.4. At first glance, it looks like we used that the Gibbs map $G: A \mapsto \mu_{A}$ is one-to-one in this proof, while we were only able to prove it in some cases in Remark 2.11. But in fact, the above proof rather implies the injectivity of $G$, as by strict convexity for all $\nu$ it can exist at most one critical point of $H_{\nu}$ on $\mathcal{Q}$.

Remark 7.5. When $\nu=\mu_{B}$ is a Gibbs measure we thus obtain

$$
\mathrm{h}_{\mathcal{X}}\left(\mu_{B}\right)=\int\left(-\log e^{N(B)(y)}\right) \mathrm{d} \mu_{B}(y)
$$

where $e^{N(B)}$ can be interpreted as a transition probability, or as the Jacobian of " $\mathrm{d} \mu / \mathrm{d} \mu$ o $T$ " (Remark 2.11). This can be used for some $(\Omega, T, \mathcal{X}(\Omega))$ to show that $\mathrm{h}_{\mathcal{X}}\left(\mu_{B}\right)$ is equal to the metric entropy $h\left(\mu_{B}\right)$; in particular this is the case for the shift $\sigma$ acting on the Bernoulli space $\mathcal{A}^{\mathbb{N}}$ with Hölder potentials (the Classical Thermodynamical Formalism in the sense of [PP90]).

In this case $\left(\sigma, \mathcal{A}^{\mathbb{N}}, \operatorname{Hol}_{\alpha}\right)$ the equality $\mathrm{h}_{\mathcal{X}}(\nu)=h(\nu)$ extends to any invariant probability $\nu$. Indeed by Theorem 9.12 in [Wal82] for any $\sigma$-invariant probability $\nu$ on the Bernoulli space, the metric entropy $h(\nu)$ satisfies

$$
h(\nu)=\inf _{A \in C^{0}\left(\mathcal{A}^{\mathbb{N}}\right)}\left\{P(A)-\int A \mathrm{~d} \nu\right\}
$$

where $P$ is the topological pressure. As topological pressure is a continuous function on the continuous potential $A$ (see Theorem 9.7 in [Wal82]) and the set of Hölder functions is dense in $C^{0}\left(\mathcal{A}^{\mathbb{N}}\right)$, the infimum above can be restricted to the Hölder potentials $A$. For Hölder potentials the pressure satisfies $P(A)=\log \lambda_{A}$ and this shows that $\mathrm{h}_{\mathcal{X}}(\nu)=h(\nu)$. Of course this reasoning applies to all cases when the topological pressure coincides with $\log \Lambda$ and the metric entropy is the Legendre dual of pressure.

The analogous results is proved for Gibbs plans in Lemma 6 in [LMMS]. An invariant probability is particular case of a Gibbs plan (see equation (1) in [LMMS]) and this provides another proof for the equality of entropies. 


\subsubsection{The pressure functionals}

We are now in a position to extend the following classical result to our general framework.

Theorem 7.6 (Gibbs measures are equilibirum states). For all $B \in \mathcal{X}(\Omega)$, we have $\operatorname{Pr}\left(\mu_{B}\right)=\log \lambda_{B}$ and $\mu_{B}$ is the unique maximizer of $P_{B}(\mu)=\mathrm{h}_{\mathcal{X}}(\mu)+\int B \mathrm{~d} \mu$ among all T-invariant probability measures.

Proof. Simply observe that

$$
P_{B}(\mu)-\log \lambda_{B}=\inf _{A \in \mathcal{X}(\Omega)} H_{\mu}(A)-H_{\mu}(B)
$$

Consider the functional $A \mapsto H_{\mu}(A)-H_{\mu}(B)$ : it takes the value 0 at $A=B$ and by Corollary 7.3 this is its infimum precisely when $\mu=\mu_{B}$. We deduce that $P_{B}\left(\mu_{B}\right)=\log \lambda_{B}$ and that for any other measure $\mu \in \mathcal{P}_{T}(\Omega), P_{B}(\mu)<\log \lambda_{B}$.

Remark 7.7. The expression $\operatorname{Pr}\left(\mu_{B}\right)=\log \lambda_{B}$ shows that $\operatorname{Pr}$ and $\mathrm{h}_{\mathcal{X}}$ are really Legendre duals one to the other, since we can now write the later $\mathrm{h}_{\mathcal{X}}(\mu)=\inf _{A} \operatorname{Pr}(A)-\int A \mathrm{~d} \mu$.

Remark 7.8. We can deduce from that result that $h_{\mathcal{X}}$ is the metric entropy and $\operatorname{Pr}$ the topological pressure whenever we know the later to be equal to $\log \Lambda$ and the former to be its Legendre dual. In particular, this holds when $T$ is the shift over a finite alphabet and $\mathcal{X}=\mathrm{Hol}_{\alpha}$, but of course in this case it is possible and more satisfactory to prove that $h_{\mathcal{X}}$ and $\operatorname{Pr}$ are the classical quantities ${ }^{6}$ and recover their interpretation in terms of eigenvalue and Legendre dual by the above.

Remark 7.9. As a particular case, the measure of maximal entropy is unique and equal to $\mu_{0}$ where 0 is the zero of $\mathcal{X}(\Omega)$. One can then describe $\mu_{0}$ as the stationary measure for the Markov chain on $\Omega$ defined by the normalized potential $N(0)$. When $T$ is $d$-toone, then $-\log d$ is obviously normalized and in the class of 0 modulo $\mathcal{C}$, so that the measure of maximal entropy is the stationary measure for the uniform random walk on backward orbits of $T$.

However, this hides some complications when points of $\Omega$ do not all have the same number of inverse images under the action of $T$ : it might then be quite difficult to express $N(0)$.

\subsection{Gradients and gradient flows}

\subsubsection{Computation of some gradients}

We can now use the metric $\langle\cdot, \cdot\rangle_{A}$ to define the gradients of the functionals $\mathrm{h}_{\mathcal{X}}$ and $P_{B}$.

Note that a weak Riemannian metric such as $\langle\cdot, \cdot\rangle_{A}$ does not give a gradient to all $C^{1}$ functionals: indeed $\langle\cdot, \cdot\rangle_{A}$ induces a continuous, one-to-one map from the tangent space

\footnotetext{
${ }^{6}$ For example one can proceed as in [LMMS15], noting that we use here the classical normalization.
} 
of $\mathcal{N}$ to its dual, but this map is not onto. ${ }^{7}$ Only those functional whose differential belong to the image of this map will have a gradient.

First, the results of Section 4 and the very definition of the metric yields that $G_{\varphi}$ : $[A] \mapsto \int \varphi \mathrm{d} \mu_{A}$ defined on the quotient $\mathcal{Q}$ has a gradient:

$$
\begin{aligned}
D\left(G_{\varphi}\right)_{A}(\zeta) & =\langle\varphi, \zeta\rangle_{A} \\
& =\langle[\varphi],[\zeta]\rangle_{[A]} \\
\nabla G_{\varphi}([A]) & =[\varphi] .
\end{aligned}
$$

Similarly, the function $A \mapsto \int \varphi \mathrm{d} \mu_{A}$ defined on $\mathcal{N}$ has a gradient at $A$, given by $D N_{A}(\varphi)$ (recall that the gradient must be a vector in $T_{A} \mathcal{N}=\operatorname{ker} \mathscr{L}_{A}$ and that $D N_{A}$ is precisely the projection on this space along $\mathcal{C})$.

Then, we consider the map $A \mapsto \mathrm{h}_{\mathcal{X}}\left(\mu_{A}\right)$. As before, we will abusively denote by $\mathrm{h}_{\mathcal{X}}$ this map, as well as its restriction to $\mathcal{N}$ and the map it induces on $\mathcal{Q}$.

From $\mathrm{h}_{\mathcal{X}}(A)=-\int N(A) \mathrm{d} \mu_{A}$, the product rule yields

$$
\begin{aligned}
D\left(\mathrm{~h}_{\mathcal{X}}\right)_{A}(\zeta) & =-\int D N_{A}(\zeta) \mathrm{d} \mu_{A}-\langle N(A), \zeta\rangle_{A} \\
& =\langle-A, \zeta\rangle_{A}
\end{aligned}
$$

since $D N_{A}(\zeta) \in \operatorname{ker} \mathscr{L}_{A} \subset \operatorname{ker} \mu_{A}$ and $\mathcal{C}=\operatorname{ker}\langle\cdot, \cdot\rangle_{A}$. This computation shows further that $\mathrm{h}_{\mathcal{X}}$ (now considered as induced on $\mathcal{Q}$ or restricted to $\mathcal{N}$ ) has a gradient:

$$
\nabla \mathrm{h}_{\mathcal{X}}([A])=-[A], \quad \text { or again } \quad \nabla \mathrm{h}_{\mathcal{X}}(A)=-D N_{A}(A) \text { when } A \in \mathcal{N} .
$$

Observing that $P_{B}(A)=\mathrm{h}_{\mathcal{X}}(A)+G_{B}(A)$ we thus proved the following.

Proposition 7.10. The maps $G_{\varphi}, \mathrm{h}_{\mathcal{X}}$ and $P_{B}$ have gradients for the weak Riemannian metric $\langle\cdot, \cdot\rangle_{A}$, given by

$$
\begin{aligned}
\nabla G_{\varphi}([A]) & =[\varphi] & \nabla G_{\varphi}(A) & =D N_{A}(\varphi) \\
\nabla \mathrm{h}_{\mathcal{X}}([A]) & =-[A] & \nabla \mathrm{h}_{\mathcal{X}}(A) & =-D N_{A}(A) \\
\nabla\left(P_{B}\right)([A]) & =[B-A] & \nabla\left(P_{B}\right)(A) & =D N_{A}(B-A)
\end{aligned}
$$

where the functionals are considered either on $\mathcal{Q}$ (left column) or $\mathcal{N}$ (right column).

\subsubsection{Gradient flow}

One particularly nice feature of the gradient of the pressure $P_{B}$ computed in Section 7.3.1 is that it straightforwardly induces a gradient flow: for all $\left[A_{0}\right] \in \mathcal{Q}$, there is a differentiable curve $\left[A_{t}\right]$ such that for all $t$

$$
\frac{\mathrm{d}}{\mathrm{d} t}\left[A_{t}\right]=\nabla\left(P_{B}\right)\left(A_{t}\right) .
$$

\footnotetext{
${ }^{7}$ If it where, by Banach's isomorphism theorem the map $\zeta \mapsto\langle\zeta, \cdot\rangle_{A}$ from $\mathcal{X}(\Omega)$ to its dual would be an isomorphism, which is equivalent to $\langle\cdot, \cdot\rangle_{A}$ being strongly positive-definite.
} 
Indeed, a solution is given by

$$
\left[A_{t}\right]=e^{-t}\left[A_{0}-B\right]+[B] .
$$

Let us give a physical interpretation when $T$ is the shift: we consider a system consisting of a $\mathbb{Z}$-lattice of particles, a potential $A_{0}$ then represents a combination of the interaction (and self-interaction) energy of the particles and of the temperature, the Gibbs measure $\mu_{A_{0}}$ is an equilibrium state (which minimizes the "free energy" $-P_{A_{0}}$ ) and represents the macroscopic state of the system at equilibrium. Assume now that this system interactions changes instantly to be now described by the potential $B$. The gradient flow above is a natural and simple model for the evolution of the macroscopic state of the system, where the systems evolves "driven" by $B$. Note that in this interpretation, the state of the system out of equilibrium is an equilibrium state for a varying potential.

Remark 7.11. Let us consider a particular case, where the interactions are constant and only the temperature changes: $A_{0}=\frac{1}{T_{0}} \varphi$ and $B=\frac{1}{T_{1}} \varphi$ for some $\varphi \in \mathcal{X}(\Omega)$; this corresponds to a system in contact with a heat bath whose temperature changes suddenly. According to our model, the system then evolves only in its temperature, as

$$
\left[A_{t}\right]=e^{-t}\left[A_{0}-B\right]+[B]=\left(e^{-t}\left(\frac{1}{T_{0}}-\frac{1}{T_{1}}\right)+\frac{1}{T_{1}}\right)[\varphi]
$$

will be proportional to $[\varphi]$ for all $t$. Note that here, $t$ should not be considered as the time as the speed of evolution of temperature would not be right. It might be possible to give a physical interpretation to the parameter $t$, or to rescale the functional $P_{B}$ and the metric in a way to obtain a physically sound evolution of the temperature.

Remark 7.12. Beware that this gradient flow really takes place on $\mathcal{Q}$ (or equivalently, on $\mathcal{N})$ : it is not defined on the whole of $\mathcal{X}(\Omega)$ because there the metric has a non-trivial kernel. Also, we cannot see this gradient flow as taking place in the set of invariant measures with the Wasserstein structure, because of Section 6: the Gibbs map is not differentiable, and when $\left(A_{t}\right)$ is a integral curve of our gradient flow, the curve $\left(\mu_{A_{t}}\right)$ is not absolutely continuous (Theorem 6.2) and in particular not a gradient flow curve in the sense of Ambrosio, Gigli and Savaré [AGS05].

\subsection{Prescribing integrals}

In this section we study how one can find Gibbs measures with prescribed values for the integrals of a given set of test functions. This is both an application of the tools we introduced here (in particular, the weak metric of Section 5 makes the proof quite easy), and a main ingredient in the proof of existence and uniqueness of equilibrium states under linear constraints.

Fix a tuple of test functions $\Phi=\left(\varphi_{1}, \ldots, \varphi_{K}\right) \in \mathcal{X}(\Omega)^{K}$; we want to study the set $\operatorname{Rot}(\Phi)$ of possible values taken by the rotation vector

$$
\operatorname{rv}(\mu)=\left(\int \varphi_{1} \mathrm{~d} \mu, \ldots, \int \varphi_{K} \mathrm{~d} \mu\right) \in \mathbb{R}^{K}
$$


where $\mu$ runs over the set $\mathcal{P}_{T}$ of $T$-invariant probability measures, and the freedom one has to prescribe the values of these integral with respect to a Gibbs measure.

It is well-known and straightforward that $\operatorname{Rot}(\Phi)$ is convex; it must also be bounded since potentials are assumed to be bounded by $(\mathrm{H} 1)$.

Observe that if the classes modulo $\mathcal{C}$ of the $\varphi_{k}$ are linearly dependent, then their integrals with respect to any invariant measure must satisfy a linear relation. Let us be more specific: if $g-g \circ T+c$ is any element of $\mathcal{C}$ and $\mu$ is any $T$-invariant probability measure, then $\int(g-g \circ T+c) \mathrm{d} \mu=c$. Therefore, if there is a non-trivial relation $\sum x_{k}\left[\varphi_{k}\right]=0$ then there are $g \in \mathcal{X}$ and $c \in \mathbb{R}$ such that $\sum x_{k} \varphi_{k}=g-g \circ T+c$ and for all $\mu \in \mathcal{P}_{T}$ we get the relation $\sum x_{k} \int \varphi_{k} \mathrm{~d} \mu=c$, constraining the vector of integrals to an affine subspace of $\mathbb{R}^{K}$. But this constraint on the rotation vector can be worked out from the $\varphi_{k}$, and one can restrict to a maximal subset of indexes $S \subset\{1, \ldots, K\}$ such that the family $\left(\left[\varphi_{k}\right]\right)_{k \in S}$ is linearly independent. Then the corresponding integrals will determine the integrals of all $\varphi_{k}$. This procedure reduces the problem to the case when the $\left[\varphi_{k}\right]$ are linearly independent, which we will always assume in the sequel.

We then get the following (which does not pretend to much originality, see [KW14] and [Jen01]; note that our proof is close to the one by Kucherenko and Wolf, but the metric $\langle\cdot, \cdot\rangle_{A}$ makes the injectivity of the Jacobian obvious and we use a differential-geometric argument to show that the map is onto).

Theorem 7.13. Let $\Phi=\left(\varphi_{1}, \ldots, \varphi_{K}\right) \in \mathcal{X}(\Omega)^{K}$ be such that the classes $\left[\varphi_{1}\right], \ldots,\left[\varphi_{K}\right]$ modulo $\mathcal{C}$ are linearly independent. Then for all $B \in \mathcal{X}(\Omega)$, the map

$$
\begin{aligned}
\mathbb{R}^{K} & \rightarrow \operatorname{int} \operatorname{Rot}(\Phi) \\
\left(a_{1}, \ldots, a_{K}\right) & \mapsto \operatorname{rv}\left(\mu_{B+a_{1} \varphi_{1}+\cdots+a_{K} \varphi_{K}}\right)
\end{aligned}
$$

is an analytic diffeomorphism; in particular $\operatorname{Rot}(\Phi)$ has non-empty interior and all its interior values are achieved by Gibbs measures.

Proof. Consider the analytic maps

$$
\begin{aligned}
I: \mathbb{R}^{K} & \rightarrow \mathcal{X}(\Omega) \\
\bar{\alpha}=\left(\alpha_{1}, \ldots, \alpha_{K}\right) & \mapsto B+\sum \alpha_{k} \varphi_{k}
\end{aligned}
$$

where $B$ is any fixed potential,

$$
\begin{aligned}
J: \mathcal{X}(\Omega) & \rightarrow \mathbb{R}^{K} \\
A & \mapsto\left(\int \varphi_{1} \mathrm{~d} \mu_{A}, \ldots, \int \varphi_{K} \mathrm{~d} \mu_{A}\right)
\end{aligned}
$$

and their composition $L=J \circ I: \mathbb{R}^{K} \rightarrow \mathbb{R}^{K}$. We also denote by $L_{k}$ the $k$-th component of $L$, i.e. $L_{k}(\bar{\alpha})=\int \varphi_{k} \mathrm{~d} \mu_{I(\bar{\alpha})}$.

The differential of $L$ is given by Sections 4 and 5 :

$$
\frac{\partial L_{k}}{\partial x_{j}}(\bar{\alpha})=\left\langle\left[\varphi_{k}\right],\left[\varphi_{j}\right]\right\rangle_{I(\bar{\alpha})} .
$$


This defines a Gram matrix, which is invertible since the $\left[\varphi_{k}\right]$ are linearly independent; it follows from the local inverse function theorem that $L$ is a local diffeomorphism.

If $B$ is any potential, this implies that $L(B)$ is in the interior of the image of $L$, in particular in the interior of $\operatorname{Rot}(\Phi)$ (which must thus be non-empty).

What we have left to prove is that $L$ is a global diffeomorphism from $\mathbb{R}^{K}$ to int $\operatorname{Rot}(\Phi)$. Since that interior is diffeomorphic to $\mathbb{R}^{K}$, a Theorem of [Gor72] reduces this to prove that $L$ is proper when its codomain is taken to be $\operatorname{int} \operatorname{Rot}(\Phi)$, i.e. that whenever a sequence $\bar{x}^{(n)}$ escapes compacts of $\mathbb{R}^{K}$, the points $L\left(\bar{x}^{(n)}\right)$ escapes the compacts of int $\operatorname{Rot}(\Phi)$. In other words, we want to prove that if $\bar{x}^{(n)} \rightarrow \infty$ and $L\left(\bar{x}^{(n)}\right)$ converges, the limit lies on $\partial \operatorname{Rot}(\Phi)$.

Now, if $\bar{x}^{(n)} \rightarrow \infty$ and $L\left(\bar{x}^{(n)}\right)$ converges, up to taking a subsequence we can assume that $\bar{x}_{(n)}=t_{n} \bar{u}+o\left(t_{n}\right)$ where $\left(t_{n}\right)$ is a diverging sequence of positive numbers, and $\bar{u}$ is a unit vector in $\mathbb{R}^{K}$ (this is simply the compactness of the unit sphere).

Observe that if $\bar{x}$ is a boundary point of $\operatorname{Rot}(\Phi)$ and $\Phi=\sum y_{k} e_{k}^{*}$ (where $\left(e_{k}^{*}\right)$ is the canonical dual basis) is a linear form of $\mathbb{R}^{K}$ whose maximum on $\operatorname{Rot}(\Phi)$ is reached at $\bar{x}$, then

$$
\Phi(\bar{x})=\max \left\{\int \sum y_{k} \varphi_{k} \mathrm{~d} \mu \mid \mu \in \mathcal{P}_{T}\right\}
$$

and reciprocally points maximizing a linear form must lie on the boundary.

Back to $L\left(\bar{x}^{(n)}\right)$, we have $I\left(\bar{x}^{(n)}\right)=t_{n} \varphi_{\bar{u}}+o\left(t_{n}\right)$ where $\varphi_{\bar{u}}=\sum u_{k} \varphi_{k}$. The variational principle tells us that $\mu_{I\left(\bar{x}^{(n)}\right)}$ maximizes $\mathrm{h}_{\mathcal{X}}(\mu)+\int\left(t_{n} \varphi_{\bar{u}}+o\left(t_{n}\right)\right) \mathrm{d} \mu$ and it follows that the accumulation points of this sequence of measures are all maximizing measures of $\varphi_{\bar{u}}$. This precisely means that the limit of $L\left(\bar{x}^{(n)}\right)$ is a boundary point, and we are done.

As a by-product of this result, we get the following.

Corollary 7.14. If $\mathcal{X}(\Omega)$ is separable, ${ }^{8}$ then the set of Gibbs measures $G(\mathcal{X}(\Omega))$ is weakly dense in $\mathcal{P}_{T}(\Omega)$.

Proof. By assumption, there is a sequence $\left(\varphi_{k}\right)_{k \in \mathbb{N}}$ of elements of $\mathcal{X}(\Omega)$ such that all continuous $f: \Omega \rightarrow \mathbb{R}$ is the uniform limit of a subsequence $\left(\varphi_{k_{i}}\right)_{i \in \mathbb{N}}$.

Let $\mu \in \mathcal{P}_{T}(\Omega)$; from Theorem 7.13 , for each $K \in \mathbb{N}$ there is a potential $A_{K} \in \mathcal{X}(\Omega)$ such that

$$
\left|\int \varphi_{k} \mathrm{~d} \mu_{A_{K}}-\int \varphi_{k} \mathrm{~d} \mu\right|<\frac{1}{K} \quad \forall k \in\{1, \ldots, K\} .
$$

Given any continuous $f: \Omega \rightarrow \mathbb{R}$ and any $\varepsilon>0$, there is some $k_{0}$ such that $\| f-$ $\varphi_{k_{0}} \|_{\infty} \leq \varepsilon$. For all $K \geq \max \left(k_{0}, \frac{1}{\varepsilon}\right)$ we thus have

$$
\begin{aligned}
\left|\int f \mathrm{~d} \mu_{A_{K}}-\int f \mathrm{~d} \mu\right| & \leq\left|\int f \mathrm{~d} \mu_{A_{K}}-\int \varphi_{k_{0}} \mathrm{~d} \mu_{A_{K}}\right|+\left|\int \varphi_{k_{0}} \mathrm{~d} \mu_{A_{K}}-\int \varphi_{k_{0}} \mathrm{~d} \mu\right| \\
& +\left|\int \varphi_{k_{0}} \mathrm{~d} \mu-\int f \mathrm{~d} \mu\right| \\
& <3 \varepsilon
\end{aligned}
$$

Letting $\varepsilon \rightarrow 0$, we see that $\int f \mathrm{~d} \mu_{A_{K}} \rightarrow \int f \mathrm{~d} \mu$, so that $\left(\mu_{A_{K}}\right)$ converges weakly to $\mu$.

\footnotetext{
${ }^{8}$ Or more generally if in (H6) the approximation can be obtained from a fixed countable subset of $\mathcal{X}$
} 


\subsection{Optimization under constraints}

Our goal here is to optimize the $P_{B}$ functionals (for example, the entropy $\mathrm{h}_{\mathcal{X}}$ ) on natural subsets of invariant measures, obtained by constraining the integrals of some functions. These questions have been considered by Jenkinson [Jen01] in the case of entropy and Kucherenko and Wolf [KW14, KW13], with somewhat different assumptions and methods. We believe that part of our claims are more explicit in some issues.

We fix as before test functions $\Phi=\left(\varphi_{1}, \ldots, \varphi_{K}\right) \in \mathcal{X}(\Omega)^{K}$ and we consider the set $\mathcal{P}_{T}[\Phi]$ of $T$-invariant measures $\mu$ such that $\int \varphi_{k} \mathrm{~d} \mu=0$ for all $k$; among them are the Gibbs measures whose normalized potential lies in

$$
\mathcal{N}[\Phi]:=\left\{A \in \mathcal{N} \mid \forall k: \int \varphi_{k} \mathrm{~d} \mu_{A}=0\right\}
$$

We will also denote by $\mathcal{Q}[\Phi]$ the set of classes $[A] \in \mathcal{Q}=\mathcal{X}(\Omega) / \mathcal{C}$ such that $A \in \mathcal{N}[\Phi]$.

With these notation, we will prove the following constrained (or "localized") version of the variational principle.

Theorem 7.15. Let $\Phi=\left(\varphi_{1}, \ldots, \varphi_{K}\right), B \in \mathcal{X}(\Omega)^{K}$ be such that the $\left[\varphi_{k}\right]$ are linearly independent, and such that 0 is an interior vector of $\operatorname{Rot}(\Phi)$. For each $B \in \mathcal{X}(\Omega)$ denote by $B_{0}$ the unique element $B_{0}=B+a_{1} \varphi_{1}+\cdots+a_{K} \varphi_{K}$ such that $\left[B_{0}\right] \in \mathcal{Q}[\Phi]$ (Theorem 7.13).

Then $\mu_{B_{0}}$ uniquely maximizes $P_{B}$ over $\mathcal{P}_{T}[\Phi]$, and the value of the maximum is $P_{B}\left(B_{0}\right)=\log \lambda_{B_{0}}$.

Proof. We simply observe that for all $\mu \in \mathcal{P}_{T}[\Phi]$ we have

$$
P_{B}(\mu)=\mathrm{h}_{\mathcal{X}}(\mu)+\int\left(B_{0}-a_{1} \varphi_{1}-\cdots-a_{K} \varphi_{K}\right) \mathrm{d} \mu=\mathrm{h}_{\mathcal{X}}(\mu)+\int B_{0} \mathrm{~d} \mu=P_{B_{0}}(\mu) .
$$

Applying Theorem 7.6 to $P_{B_{0}}$ we see that $P_{B}\left(\mu_{B_{0}}\right)=P_{B_{0}}\left(\mu_{B_{0}}\right)=\log \lambda_{B_{0}}$ is greater than $P_{B}(\mu)=P_{B_{0}}(\mu)$ whenever $\mu \neq \mu_{B_{0}}$ is in $\mathcal{P}_{T}[\Phi]$.

We can use this to recover in our setting another result from [KW14].

Corollary 7.16. Let $\Phi=\left(\varphi_{1}, \ldots, \varphi_{K}\right) \in \mathcal{X}(\Omega)$, such that the $\left[\varphi_{k}\right]$ are linearly independent and $B \in \mathcal{X}(\Omega)$, and for $w \in \operatorname{int} \operatorname{Rot}(\Phi)$ define

$$
H(w)=\sup \left\{\mathrm{h}_{\mathcal{X}}(\mu) ; \operatorname{rv}(\mu)=w\right\} .
$$

Then $H$ is a positive, analytic map.

Proof. By Theorems 7.13 we know that there are uniquely defined analytic functions $a_{k}: \operatorname{int} C \rightarrow \mathbb{R}$ such that

$$
\operatorname{rv}\left(\mu_{a_{1}(w) \varphi_{1}+\cdots+a_{K}(w) \varphi_{K}}\right)=w \quad \forall w
$$

Setting $A(w)=a_{1}(w) \varphi_{1}+\cdots+a_{K}(w) \varphi_{K}$ and applying Theorem 7.15 to $\left(\varphi_{1}-w_{1}, \ldots, \varphi_{K}-\right.$ $\left.w_{K}\right)$ we obtain

$$
H(w)=\mathrm{h}_{\mathcal{X}}\left(\mu_{A(w)}\right)=\log \Lambda(A(w))-a_{1}(w) w_{1}-\cdots-a_{K}(w) w_{K},
$$

proving the claim. 
Remark 7.17. Assume that $T$ is the shift over a finite alphabet and $\mathcal{X}=\mathrm{Hol}_{\alpha}$ (recall that $h(\mu)=\mathrm{h}_{\mathcal{X}}(\mu)$ in this case, Remark 7.5). Let $n$ be any positive integer, and let $\Phi=\left(\varphi_{1}, \ldots, \varphi_{K}\right)$ and $B$ be Hölder functions that only depends on the first $n$ coordinates, and such that $\mathcal{Q}[\Phi]$ is non empty.

Then we claim that there is a unique measure maximizing $P_{B}(\mu)$ among all elements of $\mathcal{P}_{T}[\Phi]$, and that this measure is a $(n-1)$-steps Markov measure (i.e. a Gibbs measure $\mu_{A}$ such that $N(A)$ only depends on the first $n$ coordinates). In particular, applying this to $B=0$, there is a $(n-1)$-steps Markov measure maximizing the entropy subject to any finite set of simultaneously satisfiable constraints $\int \varphi_{k} \mathrm{~d} \mu=0$ whenever the $\varphi_{k}$ are constant on cylinder of depth $n$.

Proof. The only point that does not follow immediately from Theorem 7.15 is that $\mu_{A}$ is $n$-Markov. But we know that we can take $A=B+\sum x_{k} \varphi_{k}$ for some $\left(x_{k}\right)$; notice that this $A$ might not be normalized, but is constant on each depth- $n$ cylinder.

Now, $\mathscr{L}_{A}$ preserves the subspace of $\mathcal{X}(\Omega)$ made of functions that only depend on the first $(n-1)$ coordinates. In particular, for all $N$ the function $\mathscr{L}_{A}^{N}(\mathbf{1})$ only depends on the first $(n-1)$ coordinates. Since this is a closed space, the leading eigenfunction $h_{A}$ only depends on the first $(n-1)$ coordinates, and $h_{A} \circ T$ only depends on the first $n$ coordinates.

Now $N(A)=A+\log h_{A}-\log h_{A} \circ T-\log \lambda_{A}$ only depend on the first $n$ coordinates, which precisely means that $\mu_{A}$ is $(n-1)$-steps Markov.

Let us give a couple of examples, which we will not make as general as possible but we will intentionally keep very explicit. Let $\Omega=\{0,1\}^{\mathbb{N}}, T$ be the shift and $\mathcal{X}(\Omega)$ be a space of Hölder functions for one of the usual metrics of $\Omega$. Given any finite word $\omega$, let $\omega *$ be the cylinder defined by $\omega$, i.e. the set of words starting with $\omega$.

Example 7.18. Among shift-invariant measures $\mu$ such that $\mu(0 *)=.9$, the Bernoulli measure of parameter .9 (i.e. the law of the word $\alpha_{1} \alpha_{2} \ldots$ where the $\alpha_{j}$ are i.i.d. random variables taking the value 0 with probability .9) maximizes entropy.

Indeed, from Remark 7.17 we know that there is a Bernoulli measure realizing this maximum, and the Bernoulli measure with parameter .9 is the only one to satisfy the constraint.

Example 7.19. Among shift-invariant measures $\mu$ such that $\mu(01 *)=2 \mu(11 *)$, the Markov measure associated to the transition probabilities

$$
\begin{array}{ll}
\mathbb{P}(0 \rightarrow 0)=1-a & \mathbb{P}(0 \rightarrow 1)=a \\
\mathbb{P}(1 \rightarrow 0)=\frac{2}{3} & \mathbb{P}(1 \rightarrow 1)=\frac{1}{3}
\end{array}
$$

where $a$ is the only real solution to

$$
(1-a)^{5}=\frac{4}{27} a^{2} \quad(a \simeq 0.487803)
$$

maximizes entropy. 
It is easily seen that the constraint is satisfiable by a Markov measure, in particular by a Gibbs measure, thus we can apply Remark 7.17 to $B=0, K=1$ and $\varphi=\mathbf{1}_{10 *}-2 \cdot \mathbf{1}_{11 *}$ where $\mathbf{1}_{S}$ is the indicator function of the set $S$.

The constraints easily translates into $\mathbb{P}(1 \rightarrow 0)=\frac{2}{3}$, and we define $a=\mathbb{P}(0 \rightarrow 1)$. We know that the Gibbs entropy maximizing measure is given by a potential of the form $A=x \varphi$ where $x \in \mathbb{R}$; to translate this into the transition probabilities, we only have to normalize $A$ :

$$
N(A)=x \varphi+\log h-\log h \circ T+\log \lambda
$$

where $\lambda \in \mathbb{R}$ and $h$ only depends on the first coordinates and matters only up to a multiplicative constant; we thus define $\alpha=h(0 *) / h(1 *)$. Letting $\eta=e^{x}$, we then recover the transitions probabilities as follows:

$$
\begin{aligned}
& \mathbb{P}(0 \rightarrow 0)=e^{N(A)(00 *)}=\lambda \\
& \mathbb{P}(0 \rightarrow 1)=e^{N(A)(10 *)}=\eta \alpha^{-1} \lambda \\
& \mathbb{P}(1 \rightarrow 0)=e^{N(A)(01 *)}=\alpha \lambda \\
& \mathbb{P}(1 \rightarrow 1)=e^{N(A)(11 *)}=\eta^{-2} \lambda
\end{aligned}
$$

We then have to solve the system

$$
\left\{\begin{aligned}
1-a & =\lambda \\
a & =\eta \alpha^{-1} \lambda \\
2 / 3 & =\alpha \lambda \\
1 / 3 & =\eta^{-2} \lambda
\end{aligned}\right.
$$

This will give the only $\eta$ such that $\mu_{A}$ with the above $A$ satisfies the constraint, and from Remark 7.17 we know that $\mu_{A}$ maximizes entropy under this constraint; then the corresponding value of $a$ gives the transition probability we seek. Note that, while we have some computation to do, we do not have to estimate the actual entropy of Markov measures, nor do we have to compute directly the eigendata of $\mathscr{L}_{A}$.

The above system is easily solved by substitution: $\lambda=1-a$, then $\alpha=2 /(3(1-a))$, $\eta=2 a /\left(3(1-a)^{2}\right)$ and finally the last equation yields $\left[2 a /\left(3(1-a)^{2}\right)\right]^{2}=3(1-a)$, so that $(1-a)^{5}=\frac{4}{27} a^{2}$.

\section{Explicit computations for a restricted model}

In this section we explicitly show an example of the construction of section 5 and some of its consequences. The dynamic we consider is the shift acting on the space $\{1,2\}^{\mathbb{N}}$. We choose $\mathcal{X}$ to be the space of $\alpha$-Holder functions for any $\alpha$, and denote by $\mathcal{X}_{2}$ the subset of potentials which depend only on the first two coordinates (of elements in $\{1,2\}^{\mathbb{N}}$ ). Note that we formally cannot take $\mathcal{X}_{2}$ as our full space of potentials, since it is not invariant under composition by $T$. It is easy to check that, in this setting, (H1)-(H6) are satisfied (or one can find all the details in [PP90]). 


\subsection{A positively curved metric}

If a potential $A \in \mathcal{X}_{2}$ depends just on two coordinates then we can write $A(i, j)$ for the value of $A$ evaluated on the cylinder $i j *$ (i.e. the elements of $\{1,2\}^{\mathbb{N}}$ of the type $i j \cdots$ ), and we shall identify $\mathcal{X}_{2}$ with the space of 2 by 2 real matrices. The value of $e^{A}$ is well defined on such a cylinder, and the action of the operator $\mathscr{L}_{A}$ on potentials $\varphi$ depending only on the first coordinate explicitly reads

$$
\left(\mathscr{L}_{A} \phi(1 *), \mathscr{L}_{A} \phi(2 *)\right)=(\phi(1 *) \quad \phi(2 *))\left(\begin{array}{cc}
e^{A_{11}} & e^{A_{12}} \\
e^{A_{21}} & e^{A_{22}}
\end{array}\right)
$$

We can thus think of the operator $\mathscr{L}_{A}$ as acting on a function as a left multiplication of the matrix, and we shall denote by $L$ the map

$$
L: A=\left(\begin{array}{cc}
A_{11} & A_{12} \\
A_{21} & A_{22}
\end{array}\right) \mapsto L_{A}=\left(\begin{array}{cc}
e^{A_{11}} & e^{A_{12}} \\
e^{A_{21}} & e^{A_{22}}
\end{array}\right)
$$

which exponentiate each coordinates of a matrix $A$, and identify freely $\mathscr{L}_{A}$ and $L_{A}$.

To normalize the potential, that is to find the potential $\bar{A}:=N(A)$ differing from $A$ by a coboundary and a constant such that $\mathscr{L}_{\bar{A}}(1)=1$, we can apply the Perron-Frobenius theorem ${ }^{9}$ to the matrix $L_{A}$ and solve with respect to the maximal eigenvalue and the left eigenvector, i.e. $\ell L_{A}=\lambda_{A} \ell$. After the normalization, we obtain

$$
\bar{A}(i, j)=\frac{e^{A(i, j)} \ell_{i}}{\lambda_{A} \ell_{j}}
$$

From now on, we will assume that $A$ is normalized and avoid the notation $\bar{A}$.

We observe that the set $N\left(\mathcal{X}_{2}\right)=: \mathcal{N}_{2}$ of normalized potentials depending on two coordinates is defined by the equations

$$
\left\{\begin{array}{l}
e^{A_{11}}+e^{A_{21}}=1 \\
e^{A_{12}}+e^{A_{22}}=1
\end{array}\right.
$$

so that $L\left(\mathcal{N}_{2}\right)$ is the set of 2 by 2 column stochastic matrix, denoted by $\mathcal{S}_{2}$.

To sum up, a normalized potential in $\mathcal{N}_{2}$ can be represented by the matrix of its values on cylinders, subject to a nonlinear system of constraints, or as a column stochastic matrix after coordinate-wise exponentiation. We thus obtain a natural chart $S:[0,1] \times$ $[0,1] \rightarrow \mathcal{S}_{2}$ by setting

$$
S(x, y)=\left(\begin{array}{cc}
x & 1-y \\
1-x & y
\end{array}\right)
$$

where $x, y \in(0,1)$ can be thought of as transition probabilities $\mathbb{P}[1 \rightarrow 1]$ and $\mathbb{P}[2 \rightarrow 2]$, respectively.

This parametrization has the advantage that $\mathcal{S}_{2}$ is (an open set of) an affine subspace of $M_{2,2}(\mathbb{R})$ : it has the same tangent space at each point, a basis of which is given by

$$
\frac{\partial S}{\partial x}=\left(\begin{array}{cc}
1 & 0 \\
-1 & 0
\end{array}\right) \quad \frac{\partial S}{\partial y}=\left(\begin{array}{cc}
0 & -1 \\
0 & 1
\end{array}\right) .
$$

\footnotetext{
${ }^{9}$ see for example [Gan59] for the exact statement.
} 
A tangent vector $\psi$ to $\mathcal{S}_{2}$ at $S(x, y)$ shall be written as $\left(\psi_{1}, \psi_{2}\right)$ in this basis, so that the corresponding parametrized line $\gamma$ can be expressed for $s \in \mathbb{R}$ sufficiently small by

$$
\gamma_{S(x, y), \psi}(s)=\left(\begin{array}{cc}
x+s \psi_{1} & 1-y-s \psi_{2} \\
1-x-s \psi_{1} & y+s \psi_{2}
\end{array}\right) \in \mathcal{S}_{2}
$$

The expression above is very readable, though does not allow us to compute right away the metric of $T_{A} \mathcal{N}_{2}$. However, given $A \in \mathcal{N}_{2}$ and $\xi \in T_{A} \mathscr{N}_{2}$, by Item 2 of Theorem 5.1 , we have that

$$
\langle\zeta, \zeta\rangle_{A}=\int \zeta^{2} \mathrm{~d} \mu_{A}
$$

It will thus be convenient to work both in $\mathcal{N}_{2}$ where the functional interpretation of matrices and vectors is clear, and in $\mathcal{S}_{2}$ where the Gibbs measures naturally appear. If we consider a variation $\exp \left(A_{i j}+s \zeta_{i j}\right)$ and differentiate at zero we obtain that the system

$$
\left\{\begin{array}{l}
e^{A_{11}} \zeta_{11}+e^{A_{21}} \zeta_{21}=0 \\
e^{A_{12}} \zeta_{12}+e^{A_{22}} \zeta_{22}=0
\end{array}\right.
$$

defines $T_{A} \mathcal{N}_{2} \subset \mathcal{X}_{2}$ (in particular we see that this tangent plane depends on the point $A)$. If $L_{A}=S(x, y)$ and $\psi$ corresponds to $\zeta$ in $T_{L_{A}} \mathcal{S}_{2}$, i.e. $\psi=D L_{A}(\zeta)$, it comes

$$
\left(\begin{array}{ll}
\zeta_{11} & \zeta_{12} \\
\zeta_{21} & \zeta_{22}
\end{array}\right)=\left(\begin{array}{cc}
\frac{\psi_{1}}{x} & \frac{-\psi_{2}}{1-y} \\
\frac{-\psi_{1}}{1-x} & \frac{\psi_{2}}{y}
\end{array}\right)
$$

Now the matrix $S(x, y)$ has a right eigenvector

$$
\pi=(\pi(1 *), \pi(2 *))=\left(\frac{1-y}{2-x-y}, \frac{1-x}{2-x-y}\right),
$$

which is the invariant measure on $\{1,2\}$ of the Markov chain defined by $A$, and the measures of cylinders with respect to $\mu_{A}$ are

$$
\begin{aligned}
& \mu(11 *)=\mathbb{P}[1 \rightarrow 1] \pi(1) \\
& \mu(12 *)=\mathbb{P}[2 \rightarrow 1] \pi(2) \\
& \mu(21 *)=\mathbb{P}[1 \rightarrow 2] \pi(1) \\
& \mu(22 *)=\mathbb{P}[2 \rightarrow 2] \pi(2)
\end{aligned}
$$

It is now easy to compute the metric:

$$
\begin{aligned}
\int \zeta^{2} d \mu_{A} & =\sum_{i, j} \zeta_{i, j}^{2} \mu(i j *)=\frac{\psi_{1}^{2}}{x^{2}} \frac{x(1-y)}{2-x-y}+\frac{\psi_{2}^{2}}{(1-y)^{2}} \frac{(1-y)(1-x)}{2-x-y} \\
& +\frac{\psi_{1}^{2}}{(1-x)^{2}} \frac{(1-x)(1-y)}{2-x-y}+\frac{\psi_{2}^{2}}{y^{2}} \frac{y(1-x)}{2-x-y} \\
& =\frac{1}{2-x-y}\left(\frac{1-y}{x(1-x)} \psi_{1}^{2}+\frac{1-x}{y(1-y)} \psi_{2}^{2}\right)
\end{aligned}
$$


Proposition 8.1. The restriction $g$ of the variance metric $\langle\cdot, \cdot\rangle_{A}$ to $\mathcal{N}_{2}$ is given in the chart $S$ by

$$
g_{A}=\left(\begin{array}{cc}
\frac{(1-y)}{x(1-x)(2-x-y)} & 0 \\
0 & \frac{(1-x)}{y(1-y)(2-x-y)}
\end{array}\right)
$$

(which is positive-definite for all $x, y \in(0,1) \times(0,1))$.

This means that for $L_{A}=S(x, y)$ and $\psi=D L_{A}(\zeta)$ we have $|\zeta|_{A}^{2}=\left(\begin{array}{ll}\psi_{1} & \psi_{2}\end{array}\right) g_{A}\left(\begin{array}{l}\psi_{1} \\ \psi_{2}\end{array}\right)$.

Remark 8.2. Observe that, not incidentally, by recalling the proof of 5.2 we could have computed $\langle\zeta, \zeta\rangle_{A}$ in a more roundabout way by using the equation contained there

$$
\langle\zeta, \zeta\rangle_{A}=D^{2}(\log \Lambda)_{A}(\zeta, \zeta)=D\left(G_{\zeta}\right)_{A}(\zeta)
$$

As a side effect we easily obtain that

$$
D^{2}(\log \Lambda)_{A}(\zeta, \zeta)=\frac{x(1-y)}{(1-x)(2-x-y)} \zeta_{11}^{2}+\frac{(1-x) y}{(1-y)(2-x-y)} \zeta_{22}^{2}
$$

We see for example that when $x$ or $y$ goes to 0 , the pressure becomes very flat (as opposed to very convex, i.e. its Hessian goes to zero).

From the metric tensor, we compute the curvature at each point. For simplicity, if we let $g_{A}=\left(\begin{array}{cc}E & 0 \\ 0 & G\end{array}\right)$ then we use the explicit formula for the curvature

$$
K(A)=-\frac{1}{2 \sqrt{E G}}\left\{\left(\frac{E_{y}}{\sqrt{E G}}\right)_{y}+\left(\frac{G_{x}}{\sqrt{E G}}\right)_{x}\right\} .
$$

where subscripts indicate partial derivatives with respect to the indicated variables. The expression simplifies greatly (see Section 8.3):

Corollary 8.3. The Gaussian curvature of $g$ at $A$ is given when $L_{A}=S(x, y)$ by

$$
K(A)=\frac{1}{(2-x-y)}
$$

Remark 8.4. In the case at hand, the curvature its always strictly positive. In fact, it is even bounded away from 0 , so that $\mathcal{N}_{2}$ endowed with $g$ is not complete (indeed, if $g$ where complete then the Bonnet-Myers theorem would imply that $\mathcal{N}_{2}$ is compact).

\subsection{Rescaling the metric}

We considered in the previous reasoning the Riemmanian norm $\langle\zeta, \zeta\rangle_{A}$ of a tangent vector $\zeta$ at the potential $A$ given by the asymptotic variance, as in theorem 5.1. We wonder how rescaling the metric by the entropy would effect such curvature, based on 
previous work by McMullen [McM08]. Given the eigenvector $\pi$ of the previous section (which corresponds to the eigenmeasure) the entropy is given as a function of $x, y$ by

$$
\begin{aligned}
h(x, y)= & -\frac{1-y}{2-x-y}(x \log (x)+(1-x) \log (1-x)) \\
& -\frac{1-x}{2-x-y}((1-y) \log (1-y)+y \log (y))
\end{aligned}
$$

This function is always positive on $(0,1) \times(0,1)$ and is 0 in the limit to the vertex $(0,0)$ and the edges $\{1\} \times[0,1]$ and $[0,1] \times\{1\}$ (Figure 2$)$. Note that there is a strong asymmetry between the cases $x=0$ and $x=1$ (similarly for $y$ ), as $x=1$ means the Markov chain gets stuck at the state 1 , while $x=0$ means the random walk is always repelled away from state 1 , but then can either stay at 2 or come back to 1 , leaving enough uncertainty to yield positive entropy.

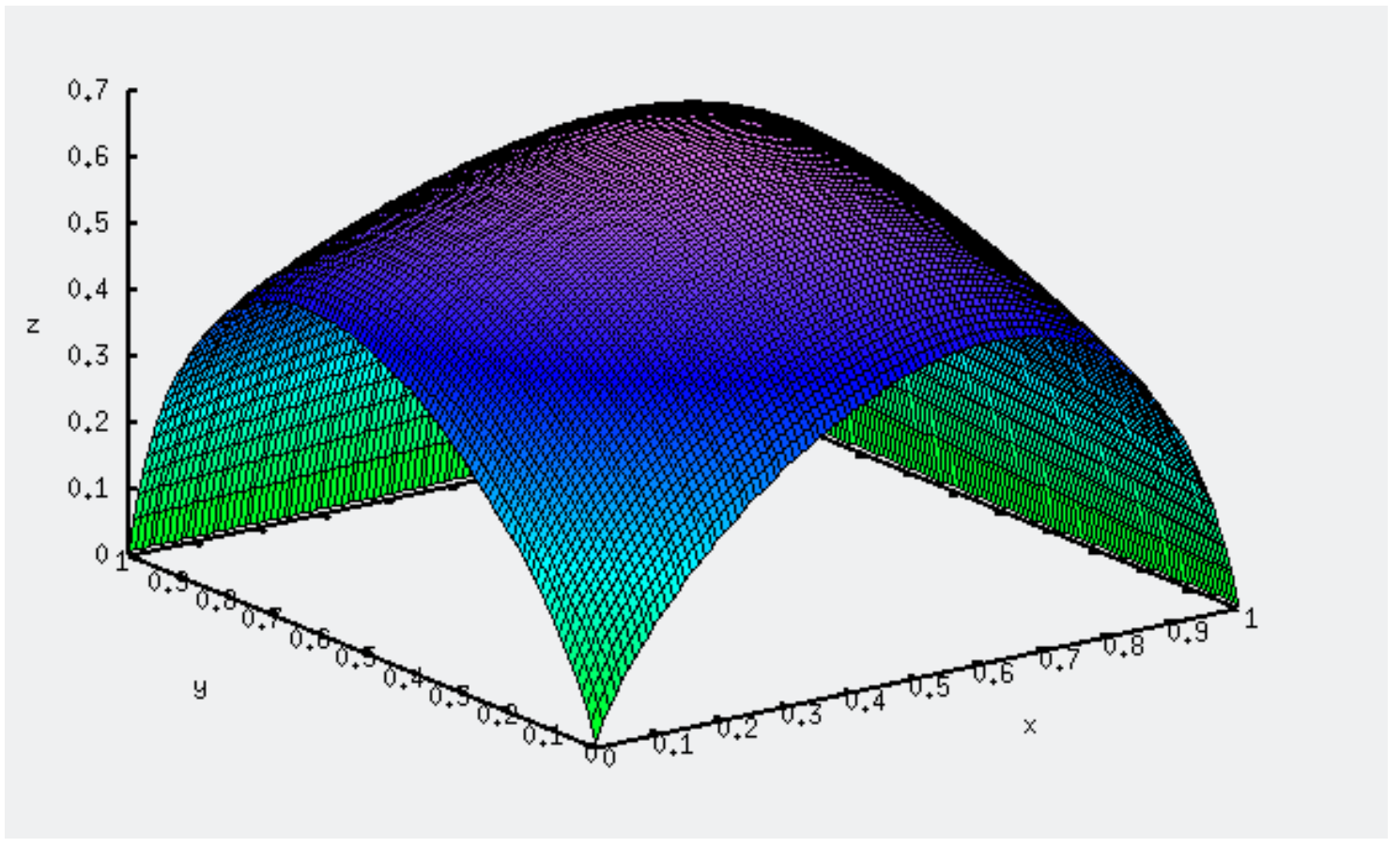

Figure 2: The entropy in $(x, y)$ coordinates.

We rescale the metric associated to the matrix $g_{A}$ of the previous section to a new $\tilde{g}_{A}$ in the interior of the square by setting $\tilde{g}_{A}=\left(\begin{array}{cc}\frac{E}{h} & 0 \\ 0 & \frac{G}{h}\end{array}\right)$ where $h$ is the entropy functional. We denote $K$ the curvature associated to the metric $g$ and $\widetilde{K}$ the one associated to $\tilde{g}$.

After a little bit of juggling with the equations, for the strictly positive function $h(x, y)$ one gets

$$
\frac{\widetilde{K}}{h}=K+\frac{1}{2 \sqrt{E G}}\left(\left(\frac{\sqrt{E}}{\sqrt{G}} \frac{h_{y}}{h}\right)_{y}+\left(\frac{\sqrt{G}}{\sqrt{E}} \frac{h_{x}}{h}\right)_{x}\right)
$$


The explicit expression of $\widetilde{K}$ is particularly long and tedious to handle. We use the software Maxima both to do the necessary symbolic manipulation and the plot of the graph (Figure 3). In the case of some subshifts related to Fuchsian groups, McMullen showed that this precise scaling of the metric identifies with the Weyl-Peterson metric on Teichmüller space, which is known to be of negative Ricci curvature. One could thus expect that $\tilde{g}$ has negative curvature, but this turns out not to be the case: $\widetilde{K}$ takes both positive and negative values.

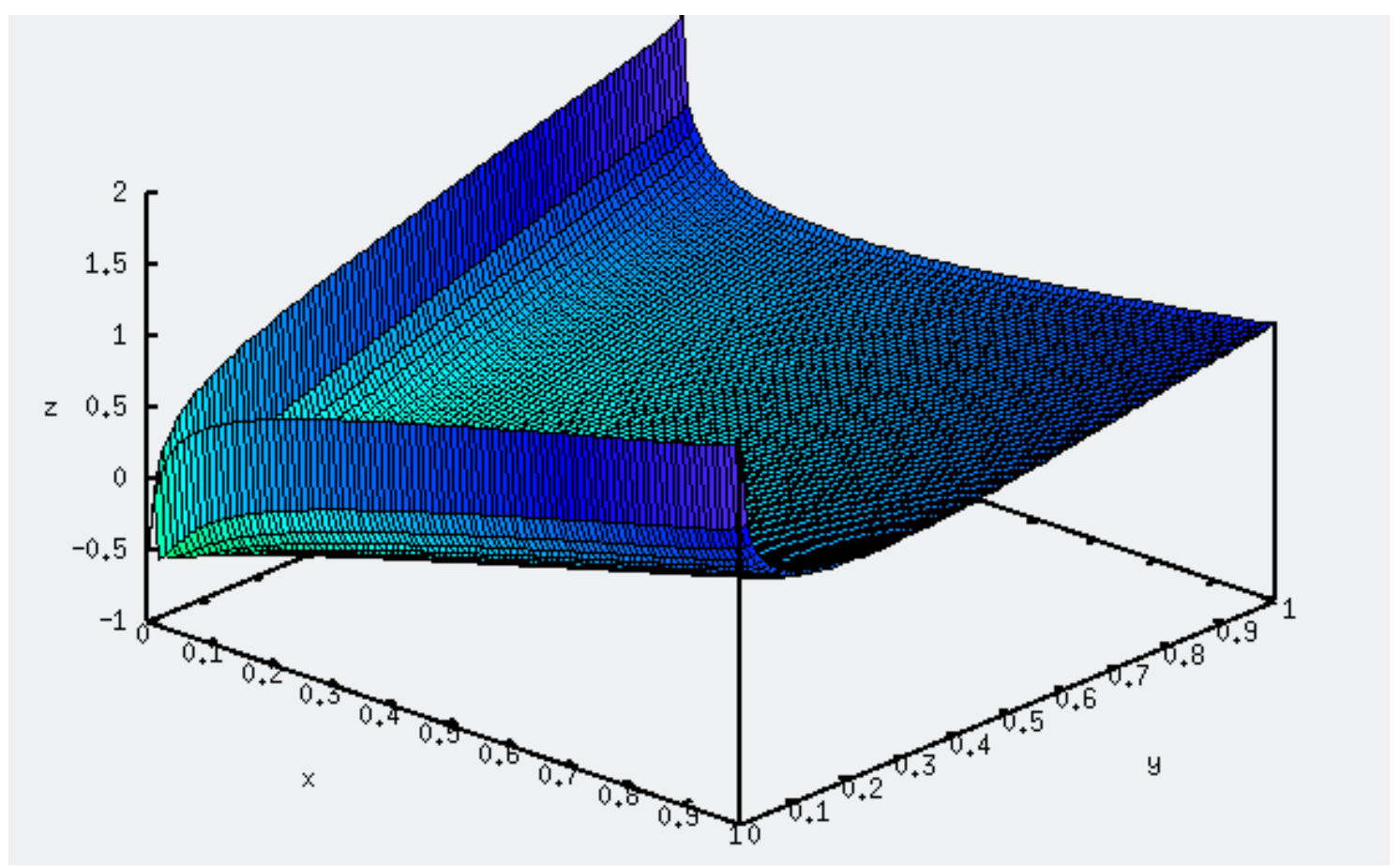

Figure 3: Curvature of the variance metric with McMullen's normalization.

\subsection{Intermediate steps}

From Section 4 of [dC76], to explicitly compute the curvature we have the followings step. Observe that

$$
E_{y}=-\frac{1}{x(2-x-y)^{2}} \quad \text { and } \quad G_{x}=-\frac{1}{y(2-x-y)^{2}} .
$$

Moreover,

$$
\sqrt{E G}=\frac{1}{\sqrt{x y}(2-x-y)} .
$$

Therefore,

$$
\frac{\partial}{\partial y}\left(\frac{E_{y}}{\sqrt{E G}}\right)=-\frac{\partial}{\partial y}\left(\frac{\sqrt{y}}{\sqrt{x}} \frac{1}{2-x-y}\right)=-\frac{1}{2 \sqrt{x y}} \frac{(2-x+y)}{(2-x-y)^{2}}
$$


and similarly

$$
\frac{\partial}{\partial x}\left(\frac{G_{x}}{\sqrt{E G}}\right)=-\frac{1}{2 \sqrt{x y}} \frac{(2+x-y)}{(2-x-y)^{2}}
$$

Finally,

$$
\begin{aligned}
\left(\frac{E_{y}}{\sqrt{E G}}\right)_{y}+\left(\frac{G_{x}}{\sqrt{E G}}\right)_{x} & =-\frac{1}{2 \sqrt{x y}} \frac{(2-x+y)}{(2-x-y)^{2}}-\frac{1}{2 \sqrt{x y}} \frac{(2+x-y)}{(2-x-y)^{2}} \\
& =-\frac{2}{\sqrt{x y}} \frac{1}{(2-x-y)^{2}}
\end{aligned}
$$

\section{References}

[AGS05] L. Ambrosio, N. Gigli et G. Savaré - Gradient flows in metric spaces and in the space of probability measures, Lectures in Mathematics ETH Zürich, Birkhäuser Verlag, Basel, 2005.

[Bal00] V. BALADI - Positive transfer operators and decay of correlations, Advanced Series in Nonlinear Dynamics, vol. 16, World Scientific Publishing Co., Inc., River Edge, NJ, 2000.

[BB00] J.-D. Benamou et Y. Brenier - "A computational fluid mechanics solution to the Monge-Kantorovich mass transfer problem", Numer. Math. 84 (2000), no. 3, p. 375-393.

[BCS15] M. Bridgeman, R. CAnARY et A. SAmbarino - "An introduction to pressure metrics on higher Teichmüller spaces", ArXiv preprint (2015), arXiv:1507.03894[math.DG].

[BCV12] T. Bomfim, A. Castro et P. VArandas - "Differentiability of thermodynamical quantities in non-uniformly expanding dynamics", ArXiv preprint (2012), arxiv:1205.5361v3[math.DS].

[BS01] L. BARREIRA et B. SAUSSOL - "Variational principles and mixed multifractal spectra", Trans. Amer. Math. Soc. 353 (2001), no. 10, p. 3919-3944 (electronic).

[BSS02] L. Barreira, B. Saussol et J. Schmeling - "Distribution of frequencies of digits via multifractal analysis", J. Number Theory 97 (2002), no. 2, p. $410-438$.

[Cha85] S. B. CHAE - Holomorphy and calculus in normed spaces, Monographs and Textbooks in Pure and Applied Mathematics, vol. 92, Marcel Dekker, Inc., New York, 1985, With an appendix by Angus E. Taylor. 
[Cli14] V. ClimenhaGA - "The thermodynamic approach to multifractal analysis", Ergodic Theory and Dynamical Systems 34 (2014), p. 1409-1450.

[dC76] M. P. DO CARMO - Differential geometry of curves and surfaces, PrenticeHall, Inc., Englewood Cliffs, N.J., 1976, Translated from the Portuguese.

[dSdSS14] E. A. DA Silva, R. R. DA Silva et R. R. Souza - "The analyticity of a generalized Ruelle's operator", Bull. Braz. Math. Soc. (N.S.) 45 (2014), no. 1 , p. 53-72.

[Gan59] F. R. Gantmacher - The theory of matrices. Vols. 1, 2, Translated by K. A. Hirsch, Chelsea Publishing Co., New York, 1959.

[Gig11] N. GigLI - "On the inverse implication of Brenier-McCann theorems and the structure of $\left(\mathscr{P}_{2}(M), W_{2}\right) "$, Methods Appl. Anal. 18 (2011), no. 2, p. 127158.

[Gor72] W. B. Gordon - "On the diffeomorphisms of Euclidean space", Amer. Math. Monthly 79 (1972), p. 755-759.

[Jen01] O. Jenkinson - "Rotation, entropy, and equilibrium states", Trans. Amer. Math. Soc. 353 (2001), no. 9, p. 3713-3739.

[Klo13] B. KLOECKNER - "Optimal transport and dynamics of expanding circle maps acting on measures", Ergodic Theory Dynam. Systems 33 (2013), no. 2, p. $529-548$.

[Klo15a] - , "Counter-examples to an infinitesimal version of the furstenberg conjecture", Ergodic Theory Dynam. Systems (2015), To appear.

[Klo15b] B. R. KLOECKNER - "A geometric study of Wasserstein spaces: ultrametrics", Mathematika 61 (2015), no. 1, p. 162-178.

[KLS14] B. Kloeckner, A. Lopes et M. Stadlbauer - "Contraction in the Wasserstein metric for some Markov chains, and applications to the dynamics of expanding maps", ArXiv preprint (2014), arxiv:1412.0848v2[math.DS].

[KW13] T. KuCherenko et C. WOLF - "Localized pressure and equilibrium states", ArXiv preprint (2013), arXiv:1310.4030 [math.DS].

[KW14] _ , "Geometry and entropy of generalized rotation sets", Israel J. Math. 199 (2014), no. 2, p. 791-829.

[LMmS] A. O. Lopes, J. K. Mengue, J. Mohr et R. R. Souza - "Entropy, pressure and duality for gibbs plans in ergodic transport", Bull. Braz. Math. Soc. (N.S.). 
[LMMS15] — , "Entropy and variational principle for one-dimensional lattice systems with a general a priori probability: positive and zero temperature", Ergodic Theory and Dynamical Systems 35 (2015), p. 1925-1961.

[Mañ90] R. MAÑÉ - "The Hausdorff dimension of horseshoes of diffeomorphisms of surfaces", Bol. Soc. Brasil. Mat. (N.S.) 20 (1990), no. 2, p. 1-24.

[McM08] C. T. MCMullen - "Thermodynamics, dimension and the Weil-Petersson metric", Invent. Math. 173 (2008), no. 2, p. 365-425.

[Ott01] F. OTTO - "The geometry of dissipative evolution equations: the porous medium equation", Comm. Partial Differential Equations 26 (2001), no. 12, p. 101-174.

[PP90] W. PARrY et M. PollicotT - "Zeta functions and the periodic orbit structure of hyperbolic dynamics", Astérisque (1990), no. 187-188, p. 268.

[PS14] M. PollicotT et R. Sharp - "A Weil-Petersson type metric on spaces of metric graphs", Geom. Dedicata 172 (2014), p. 229-244.

[Vil03] C. Villani - Topics in optimal transportation, Graduate Studies in Mathematics, vol. 58, American Mathematical Society, Providence, RI, 2003.

[Vil09] — Optimal transport, Grundlehren der Mathematischen Wissenschaften [Fundamental Principles of Mathematical Sciences], vol. 338, SpringerVerlag, Berlin, 2009, Old and new.

[Wal82] P. WALTERS - Dynamical systems and ergodic theory, Graduate Texts in Mathematics, vol. 79, Springer Verlag, 1982.

[Whi65] E. F. Whittlesey - "Analytic functions in Banach spaces", Proc. Amer. Math. Soc. 16 (1965), p. 1077-1083. 